Report no. 04/12

\title{
Time and space adaptivity for the second-order wave equation
}

\author{
by Christine Bernardi ${ }^{1}$ and Endre Süli $^{2}$
}

\begin{abstract}
The aim of this paper is to show that, for a linear second-order hyperbolic equation discretized by the backward Euler scheme in time and continuous piecewise linear finite elements in space, the adaptation of the time steps can be combined with spatial mesh adaptivity in an optimal way. We derive a priori and a posteriori error estimates which admit, as much as it is possible, the decoupling of the errors committed in the temporal and spatial discretizations.

Résumé: Le but de cet article est de montrer que, pour une équation hyperbolique modèle discrétisée par schéma d'Euler implicite en temps et éléments finis en espace, l'adaptativité des pas de temps peut se combiner avec l'adaptativité du maillage de façon optimale. Pour cela, nous prouvons des estimations d'erreur a priori et a posteriori permettant de découpler au maximum l'erreur issue de la discrétisation en temps et celle due à la discrétisation en espace.
\end{abstract}

Key words: Wave equation, backward Euler scheme, finite elements, error indicators.

June, 2004

1 Analyse Numérique, C.N.R.S. \& Université Pierre et Marie Curie, B.C. 187, 4 place Jussieu, 75252 Paris Cedex 05, France.

2 University of Oxford, Computing Laboratory, Wolfson Building, Parks Road, Oxford OX1 3QD, UK. 



\section{Introduction.}

Over the last two decades there has been considerable interest in the a posteriori error analysis of finite element approximations to partial differential equations. While for elliptic and, to a certain extent, parabolic problems, there is a fairly well-developed body of theory of a posteriori error estimation which is reviewed in detail in a number of sources (see, for example, the monographs [20], [1], [3], and the articles [7], [9], [10], [12], [13], [16], [21]), the a posteriori error analysis of finite element approximations to second-order hyperbolic problems is in a less complete state [18], [19], [2], [5], [6].

The aim of this paper is to develop the a posteriori error analysis of fully discrete approximations to a model initial-boundary-value problem for the linear second-order wave equation

$$
\partial_{t}^{2} u-\Delta u=0
$$

in a bounded connected open domain in $\mathbb{R}^{d}, d=1,2$ or 3 ; much of our analysis extends to more complicated problems.

The outline of the paper is as follows. In Section 2, we recall some key energy estimates for the wave equation. The purpose of these is to motivate the derivation of the discrete estimates which are proved in the sequel. Section 3 is devoted to the description of the time semidiscretization: this is arrived at by using a second difference quotient of $u$ on a non-uniform temporal grid to approximate $\partial_{t}^{2} u$ and sampling $\Delta u$ at the upper-most time level. On using the substitution $v=\partial_{t} u$ and rewriting the second-order wave equation as a first-order system, we show that this time discretization is equivalent to a backward Euler time discretization of the associated first-order system. By mimicking the energy analysis of Section 2, we then show that the scheme is stable, and we prove optimal $a$ priori error estimates. In Section 4, we describe the fully discrete problem where the time discretization from Section 3 is combined with a continuous piecewise affine finite element method in space. We admit non-uniform time steps and spatial meshes that vary in time. Concerning the use of variable time steps in the framework of ordinary differential equations, we refer to the monograph of Hairer, Nørsett and Wanner [15]. We prove the unconditional stability of the fully discrete scheme and perform its a priori error analysis. Section 5 is devoted to the a posteriori error analysis of the fully-discrete problem. We exhibit two types of error indicators, associated, respectively, with the temporal and the spatial discretizations. We prove upper and lower bounds for each of them. Possible extensions of our analytical result are discussed in Section 6 .

\section{Some properties of the wave equation.}

Let $\Omega$ be a bounded connected open set in $\mathbb{R}^{d}, d=1,2$ or 3 , with Lipschitz-continuous boundary $\partial \Omega$, and let $T$ be a positive real number. We consider the following initial 
boundary-value problem for the wave equation:

$$
\begin{cases}\partial_{t}^{2} u-\Delta u=0 & \text { in } \Omega \times] 0, T[, \\ u=0 & \text { on } \partial \Omega \times] 0, T[, \\ u(\cdot, 0)=u_{0} & \text { in } \Omega \text { at time } t=0, \\ \partial_{t} u(\cdot, 0)=v_{0} & \text { in } \Omega \text { at time } t=0 .\end{cases}
$$

The data are functions $u_{0}$ and $v_{0}$ defined on $\Omega$ and the unknown is the function $u$ defined on $\Omega \times] 0, T[$.

In what follows, we consider the usual Hilbertian Sobolev spaces $H^{s}(\Omega)$ for all nonnegative real numbers $s$. Note that the space $H^{0}(\Omega)$ coincides with the space $L^{2}(\Omega)$ of square-integrable functions. For simplicity, we denote its scalar product by $(\cdot, \cdot)$ and its norm by $\|\cdot\|$. As usual, $H_{0}^{1}(\Omega)$ stands for the closure in $H^{1}(\Omega)$ of the space $\mathscr{D}(\Omega)$ of infinitely differentiable functions with a compact support in $\Omega$, and it follows from the Poincaré-Friedrichs inequality that the seminorm: $v \mapsto\|\nabla v\|$ is a norm on $H_{0}^{1}(\Omega)$, equivalent to the norm of $H^{1}(\Omega)$. Its dual space is denoted by $H^{-1}(\Omega)$ and is equipped with the corresponding dual norm $\|\cdot\|_{H^{-1}(\Omega)}$. Finally, for any separable Banach space $E$, we consider the space $L^{1}(0, T ; E)$ of integrable functions on $(0, T)$ with values in $E$. For any nonnegative integer $m$, we also need the spaces $\mathscr{C}^{m}(0, T ; E)$ and $W^{m, 1}(0, T ; E)$ of continuously differentiable functions on $[0, T]$ up to the order $m$ with values in $E$ and of functions in $L^{1}(0, T ; E)$ such that all their derivatives up to order $m$ belong to $L^{1}(0, T ; E)$, respectively.

We begin with the following identity concerning the solution $u$. Note that the proofs in this section are presented in a formal manner; their purpose here is merely to motivate their discrete analogues to be considered later in the paper.

Lemma 2.1. Assume that the data $\left(u_{0}, v_{0}\right)$ belong to $H_{0}^{1}(\Omega) \times L^{2}(\Omega)$; then, each solution $u$ of problem (2.1) satisfies at time $t, 0 \leq t \leq T$,

$$
\left\|\partial_{t} u\right\|^{2}+\|\nabla u\|^{2}=\left\|v_{0}\right\|^{2}+\left\|\nabla u_{0}\right\|^{2}
$$

Proof: Taking the inner product of the first line in (2.1) with $\partial_{t} u$ and integrating by parts in the second term gives

$$
\frac{1}{2} \frac{\mathrm{d}}{\mathrm{d} t}\left(\left\|\partial_{t} u\right\|^{2}\right)+\frac{1}{2} \frac{\mathrm{d}}{\mathrm{d} t}\left(\|\nabla u\|^{2}\right)=0
$$

Integrating this last equation between 0 and $t$ gives the desired estimate.

Remark 2.2. By setting $v=\partial_{t} u$ and $U=\left(\begin{array}{l}u \\ v\end{array}\right)$, we observe that the first line in $(2.1)$ can equivalently be written

$$
\partial_{t} U-\left(\begin{array}{cc}
0 & 1 \\
\Delta & 0
\end{array}\right) U=0
$$


For a while, let us consider the more general system, with $F=\left(\begin{array}{c}f \\ g\end{array}\right)$ and $U_{0}=\left(\begin{array}{l}u_{0} \\ v_{0}\end{array}\right)$ :

$$
\begin{cases}\partial_{t} U-\left(\begin{array}{cc}
0 & 1 \\
\Delta & 0
\end{array}\right) U=F & \text { in } \Omega \times] 0, T[ \\
u=0 & \text { on } \partial \Omega \times] 0, T[, \\
U(\cdot, 0)=U_{0} & \text { in } \Omega \text { at time } t=0 .\end{cases}
$$

For any data $(f, g)$ in $L^{1}\left(0, T ; H_{0}^{1}(\Omega)\right) \times L^{1}\left(0, T ; L^{2}(\Omega)\right)$ and $\left(u_{0}, v_{0}\right)$ in $H_{0}^{1}(\Omega) \times L^{2}(\Omega)$, taking the inner product of the first line in $(2.3)$ with $W=\left(\begin{array}{c}-\Delta u \\ v\end{array}\right)$ leads to the following estimate, valid for $t, 0 \leq t \leq T$ :

$$
\left(\|v\|^{2}+\|\nabla u\|^{2}\right)^{\frac{1}{2}} \leq\left(\left\|v_{0}\right\|^{2}+\left\|\nabla u_{0}\right\|^{2}\right)^{\frac{1}{2}}+\int_{0}^{t}(\|g\|+\|\nabla f\|)(s) \mathrm{d} s
$$

A weaker estimate holds for less smooth data. Indeed, let us consider the Laplace operator which associates with any data $k$ in $H^{-1}(\Omega)$ the solution $z=\mathcal{L} k$ of the Laplace equation subject to homogeneous Dirichlet boundary condition:

$$
\begin{cases}-\Delta z=k & \text { in } \Omega \\ z=0 & \text { on } \partial \Omega\end{cases}
$$

This operator is self-adjoint and positive definite, and is an isometry from $H^{-1}(\Omega)$ onto $H_{0}^{1}(\Omega)$. Moreover, it can be seen [17, Chap. 1, Th. 12.3] that, for each $k$ in $H^{-1}(\Omega)$, the norm $\left\|\mathcal{L}^{\frac{1}{2}} k\right\|$ is equal to $\|k\|_{H^{-1}(\Omega)}$. Now, let us assume that the data $(f, g)$ belong to $L^{1}\left(0, T ; L^{2}(\Omega)\right) \times L^{1}\left(0, T ; H^{-1}(\Omega)\right)$ and $\left(u_{0}, v_{0}\right)$ in $L^{2}(\Omega) \times H^{-1}(\Omega)$. Then, taking the inner product of the first line in $(2.3)$ with $W=\left(\begin{array}{c}u \\ \mathcal{L} v\end{array}\right)$, we obtain the following estimate which is valid for all $t, 0 \leq t \leq T$ :

$$
\left(\|v\|_{H^{-1}(\Omega)}^{2}+\|u\|^{2}\right)^{\frac{1}{2}} \leq\left(\left\|v_{0}\right\|_{H^{-1}(\Omega)}^{2}+\left\|u_{0}\right\|^{2}\right)^{\frac{1}{2}}+\int_{0}^{t}\left(\|g\|_{H^{-1}(\Omega)}+\|f\|\right)(s) \mathrm{d} s .
$$

An analogue of estimate (2.6) will be used later on for proving a posteriori estimates.

To conclude this section, by combining the Cauchy-Lipschitz theorem with estimate (2.2), we easily deduce the well-posedness of system (2.1).

Proposition 2.3. For any data $\left(u_{0}, v_{0}\right)$ in $H_{0}^{1}(\Omega) \times L^{2}(\Omega)$, problem $(2.1)$ has a unique solution $u$ in $\mathscr{C}^{1}\left(0, T ; L^{2}(\Omega)\right) \cap \mathscr{C}^{0}\left(0, T ; H_{0}^{1}(\Omega)\right)$. Moreover this solution satisfies $(2.2)$.

\section{The time semidiscretization.}

Since we intend to work with nonuniform time steps, we introduce a partition of the interval $[0, T]$ into subintervals $\left[t_{n-1}, t_{n}\right], 1 \leq n \leq N$, such that $0=t_{0}<t_{1}<\cdots<t_{N}=T$. 
We denote by $\tau_{n}$ the length $t_{n+1}-t_{n}$, by $\tau$ the $N$-tuple $\left(\tau_{0}, \ldots, \tau_{N-1}\right)$ and by $|\tau|$ the maximum of the $\tau_{n}, 0 \leq n \leq N-1$.

The semidiscrete problem is then constructed in a very simple way, by applying Euler's backward scheme to system (2.3) in the case $F=0$. Thus, it consists of finding a family $\left(u^{n}\right)_{0 \leq n \leq N}$ in $H_{0}^{1}(\Omega) \times L^{2}(\Omega) \times H_{0}^{1}(\Omega)^{N-1}$ such that

$$
\left\{\begin{array}{lll}
\frac{u^{n+1}-u^{n}}{\tau_{n}}-\frac{u^{n}-u^{n-1}}{\tau_{n-1}}-\tau_{n} \Delta u^{n+1}=0 & \text { in } \Omega, & 1 \leq n \leq N-1, \\
u^{n+1}=0 & \text { on } \partial \Omega, \quad 1 \leq n \leq N-1, \\
u^{0}=u_{0} & \text { in } \Omega, \\
u^{1}=u_{0}+\tau_{0} v_{0} & \text { in } \Omega .
\end{array}\right.
$$

Assume that the data $\left(u_{0}, v_{0}\right)$ belong to $H_{0}^{1}(\Omega) \times L^{2}(\Omega)$. Then, $u^{0}$ and $u^{1}$ are defined by the third and fourth line in (3.1). Moreover, for each $n \geq 1, u^{n+1}$ is a solution of a Helmholtz-type equation, - more precisely, of the following variational problem.

Find $u^{n+1}$ in $H_{0}^{1}(\Omega)$ such that

$$
\forall w \in H_{0}^{1}(\Omega), \quad\left(u^{n+1}, w\right)+\tau_{n}^{2}\left(\nabla u^{n+1}, \nabla w\right)=\left(u^{n}+\frac{\tau_{n}}{\tau_{n-1}}\left(u^{n}-u^{n-1}\right), w\right) .
$$

By applying the Lax-Milgram lemma it is easy to verify that this problem has a unique solution. Hence, by induction on $n$, we also deduce that system (3.1) has a unique solution.

We now prove a stability estimate for the family $\left(u^{n}\right)_{0 \leq n \leq N}$, which can be thought of as the semidiscrete analogue of (2.2). It requires a further regularity property of the function $v_{0}$, but this is due to our choice of $u^{1}$.

Lemma 3.1. Assume that the data $\left(u_{0}, v_{0}\right)$ belong to $H_{0}^{1}(\Omega)^{2}$; then, each solution $\left(u^{n}\right)_{0 \leq n \leq N}$ of problem (3.1) satisfies, for $1 \leq n \leq N-1$,

$$
\left\|\frac{u^{n+1}-u^{n}}{\tau_{n}}\right\|^{2}+\left\|\nabla u^{n+1}\right\|^{2} \leq\left\|v_{0}\right\|^{2}+2\left\|\nabla u_{0}\right\|^{2}+2 \tau_{0}^{2}\left\|\nabla v_{0}\right\|^{2}
$$

Proof: Multiplying the first line of (3.1) by $\frac{u^{n+1}-u^{n}}{\tau_{n}}$ and integrating over $\Omega$ gives

$$
\left\|\frac{u^{n+1}-u^{n}}{\tau_{n}}\right\|^{2}+\left\|\nabla u^{n+1}\right\|^{2}=\left(\frac{u^{n}-u^{n-1}}{\tau_{n-1}}, \frac{u^{n+1}-u^{n}}{\tau_{n}}\right)+\left(\nabla u^{n}, \nabla u^{n+1}\right) .
$$

Thus, by using the Cauchy-Schwarz inequality, we obtain

$$
\left\|\frac{u^{n+1}-u^{n}}{\tau_{n}}\right\|^{2}+\left\|\nabla u^{n+1}\right\|^{2} \leq\left\|\frac{u^{n}-u^{n-1}}{\tau_{n-1}}\right\|^{2}+\left\|\nabla u^{n}\right\|^{2} .
$$


Applying this estimate recursively then leads to

$$
\left\|\frac{u^{n+1}-u^{n}}{\tau_{n}}\right\|^{2}+\left\|\nabla u^{n+1}\right\|^{2} \leq\left\|\frac{u^{1}-u^{0}}{\tau_{0}}\right\|^{2}+\left\|\nabla u^{1}\right\|^{2} .
$$

The desired estimate follows by using the third and fourth lines in (3.1).

Remark 3.2. In contrast with the solution $u$ to initial-boundary value problem (2.1), the regularity properties of the $u^{n}$ are easy to. There exists an $s \geq \frac{1}{2}$ such each $u^{n+1}$, $1 \leq n \leq N-1$, belongs to $H^{s+1}(\Omega)$; the maximal such value of $s$ is explicitly known when $\Omega$ is a two-dimensional polygon; in particular the maximal value of $s$ is $\geq 1$ when $\Omega$ is convex or in dimension $d=1$. In any case, the following estimate can be derived from (3.3) for $s \leq 1$ :

$$
\tau_{n}^{s}\left\|u^{n+1}\right\|_{H^{s+1}(\Omega)} \leq c\left(\left\|v_{0}\right\|^{2}+\left\|\nabla u_{0}\right\|^{2}+\tau_{0}^{2}\left\|\nabla v_{0}\right\|^{2}\right)^{\frac{1}{2}},
$$

so the norm of $u^{n+1}$ in $H^{s+1}(\Omega)$ is not bounded independently of $\tau$ under the present hypotheses on the regularity of the data.

Remark 3.3. When applying Euler's backward scheme to system (2.3), we obtain the problem consisting of finding a family $\left(U^{n}\right)_{0 \leq n \leq N}$, with $U^{n}=\left(\begin{array}{c}u^{n} \\ v^{n}\end{array}\right)$, such that

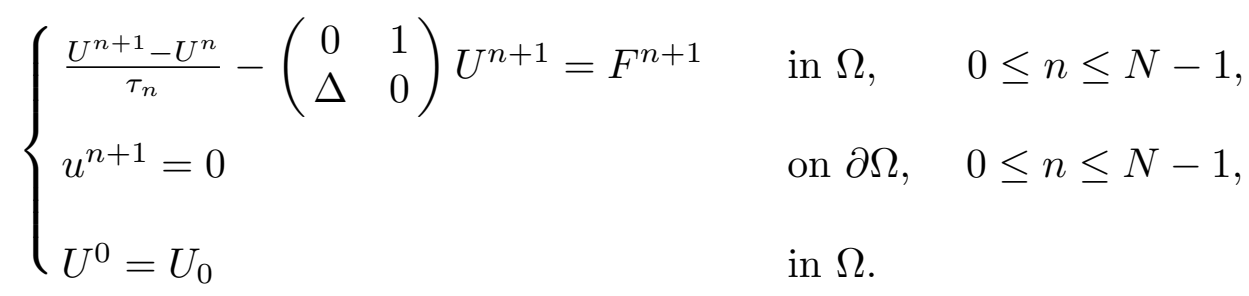

When $F^{n+1}=0$ for all $n, 0 \leq n \leq N-1$, this system coincides with problem (3.1), except at the first step when $n=0$. It would fully coincide with problem (3.1):

(i) either if the fourth line in problem (3.1), which corresponds to an explicit discretization, were replaced by the implicit equation

$$
\begin{cases}u^{1}-\tau_{0}^{2} \Delta u^{1}=u^{0}+\tau_{0} v_{0} & \text { in } \Omega \\ u^{1}=0 & \text { on } \partial \Omega\end{cases}
$$

(ii) or if system (3.6) were replaced by

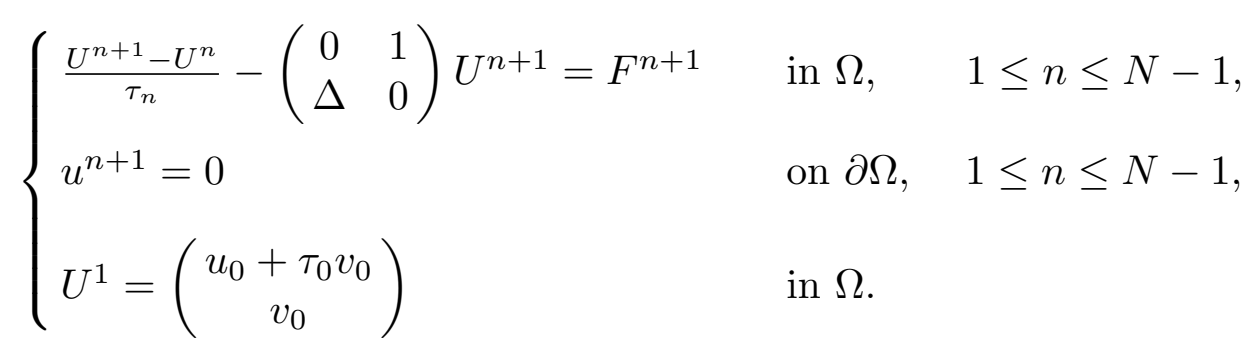

Finally, by taking the inner product of the first line of system (3.8) with $W=\left(\begin{array}{c}-\Delta u^{n+1} \\ v^{n+1}\end{array}\right)$ and using the same arguments as in the proof of (2.4), we deduce the stability estimate

$$
\left\|v^{n+1}\right\|^{2}+\left\|\nabla u^{n+1}\right\|^{2} \leq 2\left(\left\|v^{1}\right\|^{2}+\left\|\nabla u^{1}\right\|^{2}\right)+2\left(\sum_{m=1}^{n} \tau_{m}\left(\left\|g^{m+1}\right\|+\left\|\nabla f^{m+1}\right\|\right)\right)^{2},
$$


where $f^{n+1}$ and $g^{n+1}$ are the two components of $F^{n+1}$. We also note that on taking the inner product of the first line of system (3.8) with $W=\left(\begin{array}{c}u^{n+1} \\ \mathcal{L} v^{n+1}\end{array}\right)$, where $\mathcal{L}$ is the Laplace operator introduced in Section 2, leads to the following estimate in weaker norms:

$$
\begin{aligned}
& \left\|v^{n+1}\right\|_{H^{-1}(\Omega)}^{2}+\left\|u^{n+1}\right\|^{2} \\
& \quad \leq 2\left(\left\|v^{1}\right\|_{H^{-1}(\Omega)}^{2}+\left\|u^{1}\right\|^{2}\right)+2\left(\sum_{m=1}^{n} \tau_{m}\left(\left\|g^{m+1}\right\|_{H^{-1}(\Omega)}+\left\|f^{m+1}\right\|\right)\right)^{2} .
\end{aligned}
$$

The first a priori error estimate relies on estimate (3.9). Indeed, for the solution $u$ of problem (2.1) and the solution $\left(u^{n}\right)_{0 \leq n \leq N}$ of problem (3.1), let us define (with the function $v$ equal to $\left.\left.\partial_{t} u\right)\right)$

$$
E^{n}=\left(\begin{array}{c}
e_{u}^{n} \\
e_{v}^{n}
\end{array}\right), \quad \text { with } \quad e_{u}^{n}=u\left(t_{n}\right)-u^{n} \quad \text { and } \quad e_{v}^{n}=v\left(t_{n}\right)-v^{n}, \quad 1 \leq n \leq N
$$

We note that the family $\left(E^{n}\right)_{1 \leq n \leq N}$ satisfies system (3.8) with the $U^{n}$ replaced by the $E^{n}$ and the data $F^{n+1}$ replaced by the consistency errors

$$
\varepsilon_{u}^{n+1}=\frac{u\left(t_{n+1}\right)-u\left(t_{n}\right)}{\tau_{n}}-\partial_{t} u\left(t_{n+1}\right), \quad \varepsilon_{v}^{n+1}=\frac{v\left(t_{n+1}\right)-v\left(t_{n}\right)}{\tau_{n}}-\partial_{t} v\left(t_{n+1}\right) .
$$

Theorem 3.4. Assume that the solution $u$ of problem (2.1) belongs to $W^{3,1}\left(0, T ; L^{2}(\Omega)\right) \cap$ $W^{2,1}\left(0, T ; H_{0}^{1}(\Omega)\right)$; then, the following a priori error estimate holds between this solution and the solution $\left(u^{n}\right)_{0 \leq n \leq N}$ of problem (3.1), for $0 \leq n \leq N-1$ :

$$
\begin{aligned}
&\left\|\partial_{t} u\left(t_{n+1}\right)-\frac{u^{n+1}-u^{n}}{\tau_{n}}\right\|^{2}+\left\|\nabla\left(u\left(t_{n+1}\right)-u^{n+1}\right)\right\|^{2} \\
& \leq c|\tau|^{2}\left(\int_{0}^{t_{n+1}}\left(\left\|\partial_{t}^{3} u\right\|+\left\|\partial_{t}^{2} \nabla u\right\|\right)(s) \mathrm{d} s\right)^{2} .
\end{aligned}
$$

Proof: By applying estimate (3.9) to the system satisfied by the $E^{n}$, we obtain

$$
\left\|e_{v}^{n+1}\right\|^{2}+\left\|\nabla e_{u}^{n+1}\right\|^{2} \leq 2\left(\left\|e_{v}^{1}\right\|^{2}+\left\|\nabla e_{u}^{1}\right\|^{2}\right)+2\left(\sum_{m=1}^{n} \tau_{m}\left(\left\|\varepsilon_{v}^{m+1}\right\|+\left\|\nabla \varepsilon_{u}^{m+1}\right\|\right)\right)^{2} .
$$

We now evaluate the terms on the right-hand side of this inequality by using Taylor's formula

$$
\frac{w\left(t_{n+1}\right)-w\left(t_{n}\right)}{\tau_{n}}-w^{\prime}\left(t_{n+1}\right)=-\frac{1}{\tau_{n}} \int_{t_{n}}^{t_{n+1}} w^{\prime \prime}(s)\left(s-t_{n}\right) \mathrm{d} s,
$$

for bounding the terms linked to the $\varepsilon_{u}^{n}, \varepsilon_{v}^{n}$ and $e_{u}^{1}$ and a simpler formula combined with the embedding of $W^{3,1}\left(0, T ; L^{2}(\Omega)\right)$ into $\mathscr{C}^{2}\left(0, T ; L^{2}(\Omega)\right)$ for bounding the term linked to $e_{v}^{1}$. This leads to the desired estimate. 
We skip the proof of the error estimate in weaker norms, since it relies on exactly the same arguments, with (3.9) replaced by (3.10).

Corollary 3.5. Assume that the solution $u$ of problem (2.1) belongs to $W^{3,1}\left(0, T ; H^{-1}(\Omega)\right)$ $\cap W^{2,1}\left(0, T ; L^{2}(\Omega)\right)$; then, the following a priori error estimate holds between this solution and the solution $\left(u^{n}\right)_{0 \leq n \leq N}$ of problem (3.1), for $0 \leq n \leq N-1$ :

$$
\begin{aligned}
&\left\|\partial_{t} u\left(t_{n+1}\right)-\frac{u^{n+1}-u^{n}}{\tau_{n}}\right\|_{H^{-1}(\Omega)}^{2}+\left\|u\left(t_{n+1}\right)-u^{n+1}\right\|^{2} \\
& \leq c|\tau|^{2}\left(\int_{0}^{t_{n+1}}\left(\left\|\partial_{t}^{3} u\right\|_{H^{-1}(\Omega)}+\left\|\partial_{t}^{2} u\right\|\right)(s) \mathrm{d} s\right)^{2} .
\end{aligned}
$$

Since Euler's scheme is of order 1, estimates (3.13) and (3.15) are fully optimal and prove the convergence of the time semidiscretization.

\section{The space discretization.}

Henceforth, for the sake of simplicity we assume that $\Omega$ is an interval, a polygon or a polyhedron in $\mathbb{R}^{d}$ for $d=1,2,3$, respectively. At each time $t_{n}, 0 \leq n \leq N$, we introduce a regular family $\left(\mathcal{T}_{n, h}\right)_{h}$ of triangulations by closed intervals $(d=1)$, triangles $(d=2)$ or tetrahedra $(d=3)$, in the usual sense that:

- For each $h, \bar{\Omega}$ is the union of all elements of $\mathcal{T}_{n, h}$;

- For each $h$, the intersection of two different elements of $\mathcal{T}_{n, h}$, if nonempty, is a vertex, a whole edge or a whole face of the two elements;

- In dimension $d=1$, the ratio of the length $h_{K}$ of an interval $K$ to the length of any interval $K^{\prime}$ sharing a vertex with $K$ is bounded by a constant $\sigma$ independent of $K, n$ and $h$. In dimension $d \geq 2$, the ratio of the diameter $h_{K}$ of an element $K$ in $\mathcal{T}_{n, h}$ to the diameter of its inscribed circle or sphere is bounded by a constant $\sigma$ independent of $K, n$ and $h$.

In view of system (3.1) and without restriction, we choose $\mathcal{T}_{1, h}$ equal to $\mathcal{T}_{0, h}$. As usual, $h^{n}$ denotes the maximum of the diameters of the elements of $\mathcal{T}_{n, h}$. In what follows, $c$ and $c^{\prime}$ stand for generic constants independent of the $h^{n}$ and $\tau_{n}$.

The finite element space is then given by

$$
X_{n, h}=\left\{w_{h} \in H_{0}^{1}(\Omega) ; \forall K \in \mathcal{T}_{n, h}, w_{h \mid K} \in \mathcal{P}_{1}(K)\right\}
$$

where $\mathcal{P}_{1}(K)$ stands for the space of restrictions to $K$ of affine functions on $\mathbb{R}^{d}$.

To describe the discrete problem, we introduce the orthogonal projection operator $\Pi_{n, h}$ from $H_{0}^{1}(\Omega)$ onto $X_{n, h}$ : for any function $w$ in $H_{0}^{1}(\Omega), \Pi_{n, h} w$ belongs to $X_{n, h}$ and satisfies

$$
\forall z_{h} \in X_{n, h}, \quad\left(\nabla\left(w-\Pi_{n, h} w\right), \nabla z_{h}\right)=0 .
$$

Thus, the discrete problem is derived from (3.2) by a slightly modified Galerkin method. For appropriate approximations $u_{0 h}$ and $v_{0 h}$ of $u_{0}$ and $v_{0}$ in $X_{0, h}$ (note that the spaces $X_{0, h}$ and $X_{1, h}$ coincide), it reads as follows: 
Find $\left(u_{h}^{n}\right)_{0 \leq n \leq N}$ in $\prod_{n=0}^{N} X_{n, h}$ such that

$$
u_{h}^{0}=u_{0 h} \quad \text { and } \quad u_{h}^{1}=u_{0 h}+\tau_{0} v_{0 h},
$$

and, for $1 \leq n \leq N-1$,

$$
\forall w_{h} \in X_{n+1, h}, \quad\left(\frac{u_{h}^{n+1}-\Pi_{n+1, h} u_{h}^{n}}{\tau_{n}}-\frac{u_{h}^{n}-\Pi_{n, h} u_{h}^{n-1}}{\tau_{n-1}}, w_{h}\right)+\tau_{n}\left(\nabla u_{h}^{n+1}, \nabla w_{h}\right)=0 .
$$

In analogy with (3.2), it can be seen that each $u_{h}^{n+1}, 1 \leq n \leq N-1$, is a solution of the following discrete variational problem.

Find $u_{h}^{n+1}$ in $X_{n+1, h}$ such that

$$
\begin{aligned}
\forall w_{h} \in X_{n+1, h}, \quad\left(u_{h}^{n+1}, w_{h}\right)+\tau_{n}^{2} & \left(\nabla u_{h}^{n+1}, \nabla w_{h}\right) \\
& =\left(\left(\Pi_{n+1, h} u_{h}^{n}+\frac{\tau_{n}}{\tau_{n-1}}\left(u_{h}^{n}-\Pi_{n, h} u_{h}^{n-1}\right), w_{h}\right) .\right.
\end{aligned}
$$

Thus, it follows from the Lax-Milgram lemma that system (4.3) - (4.4) has a unique solution.

Remark 4.1. The introduction of the operators $\Pi_{n, h}$ is motivated by the fact that, in contrast with standard fixed-grid finite element discretizations of the wave equation, here we have chosen to work with different meshes at each time step. Note however that, if $\mathcal{T}_{n+1, h}$ is only refined with respect to $\mathcal{T}_{n, h}$, i.e. if each element of $\mathcal{T}_{n+1, h}$ is contained in an element of $\mathcal{T}_{n, h}$, then $X_{n+1, h}$ contains $X_{n, h}$, so that $\Pi_{n+1, h} u_{h}^{n}$ is equal to $u_{h}^{n}$.

We now establish a stability property for the family $\left(u_{h}^{n}\right)_{0 \leq n \leq N}$. The necessity of introducing the $\Pi_{n, h}$ is brought to light in the proof.

Lemma 4.2. Let us assume that the data $\left(u_{0 h}, v_{0 h}\right)$ belong to $X_{0, h}^{2}$; then, each solution $\left(u_{h}^{n}\right)_{0 \leq n \leq N}$ of problem (4.3) - (4.4) satisfies, for $1 \leq n \leq N-1$,

$$
\left\|\frac{u_{h}^{n+1}-\Pi_{n+1, h} u_{h}^{n}}{\tau_{n}}\right\|^{2}+\left\|\nabla u_{h}^{n+1}\right\|^{2} \leq\left\|v_{0 h}\right\|^{2}+2\left\|\nabla u_{0 h}\right\|^{2}+2 \tau_{0}^{2}\left\|\nabla v_{0 h}\right\|^{2} .
$$

Proof: By taking $w_{h}$ equal to $\frac{u_{h}^{n+1}-\Pi_{n+1, h} u_{h}^{n}}{\tau_{n}}$ (which belongs to $\left.X_{n+1, h}\right)$ in (4.4), we obtain

$$
\begin{aligned}
\left\|\frac{u_{h}^{n+1}-\Pi_{n+1, h} u_{h}^{n}}{\tau_{n}}\right\|^{2}+ & \left\|\nabla u_{h}^{n+1}\right\|^{2} \\
& =\left(\frac{u_{h}^{n}-\Pi_{n, h} u_{h}^{n-1}}{\tau_{n-1}}, \frac{u_{h}^{n+1}-\Pi_{n+1, h} u_{h}^{n}}{\tau_{n}}\right)+\left(\nabla u_{h}^{n+1}, \nabla \Pi_{n+1, h} u_{h}^{n}\right) .
\end{aligned}
$$

Thanks to the definition (4.2) of the operator $\Pi_{n+1, h}$ and since $u_{h}^{n+1}$ belongs to $X_{n+1, h}$, we have

$$
\left(\nabla u_{h}^{n+1}, \nabla \Pi_{n+1, h} u_{h}^{n}\right)=\left(\nabla u_{h}^{n+1}, \nabla u_{h}^{n}\right) .
$$


Hence, by using the Cauchy-Schwarz inequality, we obtain

$$
\left\|\frac{u_{h}^{n+1}-\Pi_{n+1, h} u_{h}^{n}}{\tau_{n}}\right\|^{2}+\left\|\nabla u_{h}^{n+1}\right\|^{2} \leq\left\|\frac{u_{h}^{n}-\Pi_{n, h} u_{h}^{n-1}}{\tau_{n-1}}\right\|^{2}+\left\|\nabla u_{h}^{n}\right\|^{2} .
$$

Applying this inequality recursively leads to

$$
\left\|\frac{u_{h}^{n+1}-\Pi_{n+1, h} u_{h}^{n}}{\tau_{n}}\right\|^{2}+\left\|\nabla u_{h}^{n+1}\right\|^{2} \leq\left\|\frac{u_{h}^{1}-\Pi_{1, h} u_{h}^{0}}{\tau_{0}}\right\|^{2}+\left\|\nabla u_{h}^{1}\right\|^{2} .
$$

Since the triangulations $\mathcal{T}_{0, h}$ and $\mathcal{T}_{1, h}$ coincide, $\Pi_{1, h} u_{h}^{0}$ is equal to $u_{h}^{0}$. This gives the desired estimate.

We conclude this section by proving an a priori error estimate between the solutions $\left(u^{n}\right)_{0 \leq n \leq N}$ and $\left(u_{h}^{n}\right)_{0 \leq n \leq N}$. We recall that there exists a Clément type operator $\mathcal{R}_{n, h}$ (see [11]) which maps $H_{0}^{1}(\Omega)$ into $X_{n, h}$ and satisfies the following estimates (see [8, Chap. IX, Th. 3.11]): for all $K$ in $\mathcal{T}_{n, h}$ and any function $v$ in $H^{s+1}(\Omega) \cap H_{0}^{1}(\Omega), 0 \leq s \leq 1$,

$$
\left\|\nabla\left(v-\mathcal{R}_{n, h} v\right)\right\|_{L^{2}(K)^{2}}+h_{K}^{-1}\left\|v-\mathcal{R}_{n, h} v\right\|_{L^{2}(K)} \leq c h_{K}^{s}\|v\|_{H^{s+1}\left(\Delta_{K}\right)},
$$

where $\Delta_{K}$ stands for the union of elements of $\mathcal{T}_{n, h}$ which have a nonempty intersection with $K$. The same estimates hold for the Lagrange interpolation operator $\mathcal{I}_{n, h}$, however with the restriction $s>\frac{d}{2}-1$ since this operator is only defined on continuous functions. We now state the analogous properties of the operator $\Pi_{n, h}$ on the whole domain, and present a sketch of their proof.

Lemma 4.3. There exists a real number $\sigma_{\Omega}, \frac{1}{2}<\sigma_{\Omega} \leq 1$, such that the following estimates hold for any function $v$ in $H^{s+1}(\Omega) \cap H_{0}^{1}(\Omega), 0 \leq s \leq 1$ :

$$
\left\|\nabla\left(v-\Pi_{n, h} v\right)\right\|+\left(h^{n}\right)^{-\sigma_{\Omega}}\left\|v-\Pi_{n, h} v\right\| \leq c\left(h^{n}\right)^{s}\|v\|_{H^{s+1}(\Omega)} .
$$

The parameter $\sigma_{\Omega}$ is equal to 1 either in dimension $d=1$ or when $\Omega$ is convex, and is $<\frac{\pi}{\omega}$ when $\Omega$ is a nonconvex polygon with largest angle $\omega$.

Proof: The first estimate in (4.8) is easily derived from the definition (4.2) of the operator $\Pi_{n, h}$ and estimate (4.7). The second estimate relies on a duality argument: we solve a Laplace equation with data $v-\Pi_{n, h} v$ and homogeneous Dirichlet boundary condition on $\partial \Omega$; recall that the solution $w$ to this problem belongs to $H^{\sigma_{\Omega}+1}(\Omega)$ for the values of the parameter $\sigma_{\Omega}$ introduced above, with the norm in this space smaller than a constant times $\left\|v-\Pi_{n, h} v\right\|$, and use the formula

$$
\left\|v-\Pi_{n, h} v\right\|^{2}=\int_{\Omega} \nabla\left(v-\Pi_{n, h} v\right) \cdot \nabla\left(w-\Pi_{n, h} w\right) \mathrm{d} \boldsymbol{x}
$$

together with the first part of (4.8).

Theorem 4.4. Assume that the solution $\left(u^{n}\right)_{0 \leq n \leq N}$ of problem (3.1) belongs to the space $H^{s+1}(\Omega)^{N+1}, 0 \leq s \leq 1$, and that the initial data $\left(u_{0 h}, v_{0 h}\right)$ are defined by

$$
u_{0 h}=\Pi_{0, h} u_{0}, \quad v_{0 h}=\Pi_{0, h} v_{0}
$$


then, the following a priori error estimate holds between this solution and the solution $\left(u_{h}^{n}\right)_{0 \leq n \leq N}$ of problem (4.3) - (4.4), for $0 \leq n \leq N-1$ :

$$
\begin{aligned}
& \left\|\frac{\left(u^{n+1}-u_{h}^{n+1}\right)-\left(u^{n}-\Pi_{n+1, h} u_{h}^{n}\right)}{\tau_{n}}\right\|^{2}+\left\|\nabla\left(u^{n+1}-u_{h}^{n+1}\right)\right\|^{2} \\
& \leq c\left(\left(h^{n+1}\right)^{2 s}\left\|u^{n+1}\right\|_{H^{s+1}(\Omega)}^{2}\right. \\
& \left.\quad+\left(\sum_{m=0}^{n} \tau_{m}^{-1}\left(\max \left\{h^{m}, h^{m+1}\right\}\right)^{\sigma_{\Omega}+s} \sum_{k=0}^{1}\left\|u^{m+1-k}\right\|_{H^{s+1}(\Omega)}\right)^{2}\right) .
\end{aligned}
$$

Proof: We set

$$
e_{h}^{n}=u_{h}^{n}-\Pi_{n, h} u^{n}, \quad \tilde{e}_{h}^{n}=u^{n}-\Pi_{n, h} u^{n}, \quad \tilde{e}_{h}^{n *}=u^{n}-\Pi_{n+1, h} \Pi_{n, h} u^{n} .
$$

By combining problems (3.1) and (4.4), and using the orthogonality property (4.2), we have, for any $w_{h}$ in $X_{n+1, h}$,

$$
\begin{aligned}
\left(\frac{e_{h}^{n+1}-\Pi_{n+1, h} e_{h}^{n}}{\tau_{n}}-\frac{e_{h}^{n}-\Pi_{n, h} e_{h}^{n-1}}{\tau_{n-1}}, w_{h}\right)+\tau_{n}\left(\nabla e_{h}^{n+1}, \nabla w_{h}\right) \\
=\left(\frac{\tilde{e}_{h}^{n+1}-\tilde{e}_{h}^{n *}}{\tau_{n}}-\frac{\tilde{e}_{h}^{n}-\tilde{e}_{h}^{n-1 *}}{\tau_{n-1}}, w_{h}\right)+\tau_{n}\left(\nabla \tilde{e}_{h}^{n+1}, \nabla w_{h}\right) \\
=\left(\frac{\tilde{e}_{h}^{n+1}-\tilde{e}_{h}^{n *}}{\tau_{n}}-\frac{\tilde{e}_{h}^{n}-\tilde{e}_{h}^{n-1 *}}{\tau_{n-1}}, w_{h}\right) .
\end{aligned}
$$

We now set

$$
Z^{n+1}=\left(\left\|\frac{e_{h}^{n+1}-\Pi_{n+1, h} e_{h}^{n}}{\tau_{n}}\right\|^{2}+\left\|\nabla e_{h}^{n+1}\right\|^{2}\right)^{\frac{1}{2}}, \quad E^{n+1}=\left\|\frac{\tilde{e}_{h}^{n+1}-\tilde{e}_{h}^{n *}}{\tau_{n}}\right\| .
$$

By taking $w_{h}=\frac{e_{h}^{n+1}-\Pi_{n+1, h} e_{h}^{n}}{\tau_{n}}$, we derive from the same arguments as in the proof of Lemma 4.2 that

$$
\left(Z^{n+1}\right)^{2} \leq Z^{n} Z^{n+1}+\left(E^{n}+E^{n+1}\right) Z^{n+1},
$$

whence

$$
Z^{n+1} \leq Z^{n}+\left(E^{n}+E^{n+1}\right)
$$

Summing over $n$ leads to

$$
Z^{n+1} \leq Z^{1}+2 \sum_{m=1}^{n+1} E^{m} .
$$

It follows from the choice (4.3) and (4.10) of the initial conditions and the fact that the triangulations $\mathcal{T}_{0, h}$ and $\mathcal{T}_{1, h}$ coincide, we have

$e_{h}^{0}=u_{h}^{0}-\Pi_{0, h} u^{0}=\Pi_{0, h} u_{0}-\Pi_{0, h} u_{0}=0 ; \quad e_{h}^{1}=u_{h}^{1}-\Pi_{1, h} u^{1}=e_{h}^{0}+\tau_{0}\left(\Pi_{0, h} v_{0}-\Pi_{0, h} v_{0}\right)=0$, 
so that $Z^{1}$ is zero. Taking the square of the previous inequality and using the triangle inequalities

$$
\left\|\frac{\left(u^{n+1}-u_{h}^{n+1}\right)-\left(u^{n}-\Pi_{n+1, h} u_{h}^{n}\right)}{\tau_{n}}\right\| \leq\left\|\frac{\tilde{e}_{h}^{n+1}-\tilde{e}_{h}^{n *}}{\tau_{n}}\right\|+\left\|\frac{e_{h}^{n+1}-\Pi_{n+1, h} e_{h}^{n}}{\tau_{n}}\right\|,
$$

and

$$
\left\|\nabla\left(u^{n+1}-u_{h}^{n+1}\right)\right\| \leq\left\|\nabla \tilde{e}^{n+1}\right\|+\left\|\nabla e_{h}^{n+1}\right\|
$$

imply that the left-hand side of (4.11) is bounded by

$$
2\left\|\nabla\left(u^{n+1}-\Pi_{n+1, h} u^{n+1}\right)\right\|^{2}+6\left(\sum_{m=1}^{n+1} E^{m}\right)^{2} .
$$

On bounding this last quantity using (4.8) and the triangle inequality

$E^{n+1} \leq\left\|\left(I d-\Pi_{n+1, h}\right)\left(\frac{u^{n+1}-u^{n}}{\tau_{n}}\right)\right\|+\left\|\left(I d-\Pi_{n, h}\right) \frac{u^{n}}{\tau_{n}}\right\|+\left\|\left(I d-\Pi_{n+1, h}\right)\left(I d-\Pi_{n, h}\right) \frac{u^{n}}{\tau_{n}}\right\|$,

we conclude the proof.

We make some further comments on estimate (4.11).

(i) In practical situations, the discrete initial conditions (4.10) are replaced by

$$
u_{0 h}=\mathcal{I}_{0, h} u_{0}, \quad v_{0 h}=\mathcal{I}_{0, h} v_{0} .
$$

This leads to the same error estimates as before, except that additional regularity requirements have to be imposed on the initial data $\left(u_{0}, v_{0}\right)$.

(ii) Under the assumption that the expressions $\left\|u^{n}\right\|_{H^{s+1}(\Omega)}, 0 \leq n \leq N$, are bounded, independent of $n$, estimate (4.11) proves the convergence of the method when the quantity

$$
\left(h^{n+1}\right)^{s}+\sum_{m=0}^{n} \tau_{m}^{-1}\left(\max \left\{h^{m}, h^{m+1}\right\}\right)^{\sigma_{\Omega}+s}
$$

tends to zero. This requires that the $h^{n}$ are small enough in comparison with $\tau_{n}$. A simple sufficient condition for retaining the optimality of the spatial approximation error would be to demand that

$$
\max \left\{h^{m}, h^{m+1}\right\} \leq C \tau_{m}^{2 / \sigma_{\Omega}} .
$$

(iii) In dimension $d=1$ or when $\Omega$ is convex, when combining (4.11) with the regularity property (3.5), we deduce that the right-hand side of (4.11) is bounded above by

$$
c\left(u_{0}, v_{0}\right)\left(\tau_{n}^{-2}\left(h^{n+1}\right)^{2}+\left(\sum_{m=0}^{n} \tau_{m}^{-2}\left(\max \left\{h^{m}, h^{m+1}\right\}\right)^{2}\right)^{2}\right)
$$

where the constant $c\left(u_{0}, v_{0}\right)$ is a constant multiple of the right-hand side of (3.5). Hence, the conditions for convergence, under the assumed (limited) regularity hypotheses on the initial data, are made explicit here. 
(iv) When combining estimates (3.13) and (4.11), we obtain an a priori estimate for the fully discrete scheme.

\section{A posteriori analysis of the discretization.}

We describe two families of refinement indicators and prove upper and lower bounds on the error, focusing first on the temporal and then on the spatial discretisation.

\section{THE TIME DISCRETIZATION}

For each $n, 1 \leq n \leq N-1$, the refinement indicator is defined by

$$
\eta_{n}=\tau_{n}\left\|\nabla\left(u_{h}^{n+1}-u_{h}^{n}\right)\right\|+\tau_{n}\left\|\frac{u_{h}^{n+1}-\Pi_{n+1, h} u_{h}^{n}}{\tau_{n}}-\frac{u_{h}^{n}-\Pi_{n, h} u_{h}^{n-1}}{\tau_{n-1}}\right\| .
$$

We refer to [16] for the original idea to employ indicators of this type and to [7] for their use in the a posteriori analysis of finite element approximations to the heat equation. We note that $\eta_{n}$ is very easy to compute once the discrete solutions $u_{h}^{n-1}, u_{h}^{n}$ and $u_{h}^{n+1}$ are known. Our aim here is to derive a posteriori bounds on the error in terms of the $\eta_{n}$.

As usual, the a posteriori analysis of the time discretization relies on an appropriate residual equation. In order to define this, let $u_{\tau}$ denote the function which is continuous, affine on each interval $\left[t_{n-1}, t_{n}\right], 1 \leq n \leq N$, and equal to $u^{n}$ at $t_{n}, 0 \leq n \leq N$. Similarly let $v_{\tau}$ denote the function which is continuous, affine on each interval $\left[t_{n-1}, t_{n}\right], 1 \leq n \leq N$, and equal to $v^{n}=\frac{u^{n}-u^{n-1}}{\tau_{n-1}}$ at $t_{n}, 1 \leq n \leq N$, and to $v_{0}$ at $t_{0}=0$. Setting $v=\partial_{t} u$, the residual system, with $U=\left(\begin{array}{l}u \\ v\end{array}\right)$ and $U_{\tau}=\left(\begin{array}{c}u_{\tau} \\ v_{\tau}\end{array}\right)$, is defined as follows

$$
\begin{cases}\partial_{t}\left(U-U_{\tau}\right)-\left(\begin{array}{cc}
0 & 1 \\
\Delta & 0
\end{array}\right)\left(U-U_{\tau}\right)=\left(\begin{array}{l}
R_{u} \\
R_{v}
\end{array}\right) & \text { in } \Omega \times] 0, T[, \\
u-u_{\tau}=0 & \text { on } \partial \Omega \times] 0, T[, \\
\left(U-U_{\tau}\right)(\cdot, 0)=0 & \text { in } \Omega \text { at time } t=0,\end{cases}
$$

where the residual quantities $R_{u}$ in $L^{1}\left(0, T ; L^{2}(\Omega)\right)$ and $R_{v}$ in $L^{1}\left(0, T ; H^{-1}(\Omega)\right)$ are affine functions on each interval $\left[t_{n},, t_{n+1}\right], 1 \leq n \leq N-1$, equal to

$$
\begin{aligned}
R_{u}(\cdot, t)= & \begin{cases}v_{\tau}-v^{n+1}, & \text { if } t_{n} \leq t \leq t_{n+1}, 1 \leq n<N-1, \\
0, & \text { if } 0 \leq t<t_{1},\end{cases} \\
& R_{v}(\cdot, t)= \begin{cases}\Delta u_{\tau}-\Delta u^{n+1}, & \text { if } t_{n} \leq t \leq t_{n+1}, 1 \leq n<N-1, \\
\Delta u_{\tau}, & \text { if } 0 \leq t<t_{1} .\end{cases}
\end{aligned}
$$

We are now in a position to prove our first a posteriori error estimate. 
Proposition 5.1. The following a posteriori error estimate holds between the solution $u$ of problem (2.1) and the solution $\left(u^{n}\right)_{0 \leq n \leq N}$ of problem (3.1), for all $t_{n+1}, 0 \leq n \leq N-1$ :

$$
\begin{aligned}
& \left\|\left(\partial_{t} u\right)\left(t_{n+1}\right)-\frac{u^{n+1}-u^{n}}{\tau_{n}}\right\|_{H^{-1}(\Omega)}+\left\|u\left(t_{n+1}\right)-u^{n+1}\right\| \\
& \leq c\left(\sum_{m=1}^{n} \eta_{m}+\tau_{0}\left\|\nabla u_{0}\right\|+\tau_{0}^{2}\left\|\nabla v_{0}\right\|\right. \\
& \quad+\sum_{m=1}^{n} \tau_{m} \sum_{k=0}^{1}\left\|\nabla\left(u^{m+1-k}-u_{h}^{m+1-k}\right)\right\| \\
& \left.\quad+\sum_{m=1}^{n} \tau_{m} \sum_{k=0}^{1}\left\|\frac{\left(u^{m+1-k}-u_{h}^{m+1-k}\right)-\left(u^{m-k}-\Pi_{m+1-k, h} u_{h}^{m-k}\right)}{\tau_{m-k}}\right\|\right) .
\end{aligned}
$$

Proof: We use the same arguments and the same notation as in the proof of estimate (2.6). On taking the inner product of (5.2) with $\left(\begin{array}{c}u-u_{\tau} \\ \mathcal{L}\left(v-v_{\tau}\right)\end{array}\right)$ and setting

$$
Z(t)=\left(\left\|\left(u-u_{\tau}\right)(\cdot, t)\right\|^{2}+\left\|\left(v-v_{\tau}\right)(\cdot, t)\right\|_{H^{-1}(\Omega)}^{2}\right)^{\frac{1}{2}}
$$

we obtain

$$
\begin{aligned}
\frac{1}{2} \frac{\mathrm{d} Z^{2}}{\mathrm{~d} t}=\left(R_{u}, u-u_{\tau}\right) & +\left(R_{v}, \mathcal{L}\left(v-v_{\tau}\right)\right) \\
& \leq\left\|R_{u}(\cdot, t)\right\|\left\|\left(u-u_{\tau}\right)(\cdot, t)\right\|+\left\|R_{v}(\cdot, t)\right\|_{H^{-1}(\Omega)}\left\|\left(v-v_{\tau}\right)(\cdot, t)\right\|_{H^{-1}(\Omega)} \\
& \leq\left(\left\|R_{u}(\cdot, t)\right\|^{2}+\left\|R_{v}(\cdot, t)\right\|_{H^{-1}(\Omega)}^{2}\right)^{\frac{1}{2}} Z
\end{aligned}
$$

whence

$$
\frac{\mathrm{d} Z}{\mathrm{~d} t} \leq\left(\left\|R_{u}(\cdot, t)\right\|^{2}+\left\|R_{v}(\cdot, t)\right\|_{H^{-1}(\Omega)}^{2}\right)^{\frac{1}{2}} \leq\left\|R_{u}(\cdot, t)\right\|+\left\|R_{v}(\cdot, t)\right\|_{H^{-1}(\Omega)} .
$$

Integrating this last inequality between 0 and $t_{n+1}$ and noting that $Z(0)$ is equal to zero, we obtain

$$
Z\left(t_{n+1}\right) \leq \int_{0}^{t_{n+1}}\left(\left\|R_{u}(\cdot, s)\right\|+\left\|R_{v}(\cdot, s)\right\|_{H^{-1}(\Omega)}\right) \mathrm{d} s .
$$

We note that $Z\left(t_{n+1}\right)$ is larger than $\frac{1}{\sqrt{2}}$ times the left-hand side of (5.3). Next, to bound the integral of $\left\|R_{v}\right\|_{H^{-1}(\Omega)}$ on each interval $] t_{m}, t_{m+1}[, 1 \leq m \leq n$, we observe that

$$
u_{\tau}(\cdot, s)=u^{m+1}-\frac{u^{m+1}-u^{m}}{\tau_{m}}\left(t_{m+1}-s\right), \quad t_{m} \leq s \leq t_{m+1},
$$

whence

$$
\int_{t_{m}}^{t_{m+1}}\left\|R_{v}(\cdot, s)\right\|_{H^{-1}(\Omega)} \mathrm{d} s=\left\|\nabla\left(u^{m+1}-u^{m}\right)\right\| \int_{t_{m}}^{t_{m+1}} \frac{t_{m+1}-s}{\tau_{m}} \mathrm{~d} s=\frac{\tau_{m}}{2}\left\|\nabla\left(u^{m+1}-u^{m}\right)\right\|,
$$


and we conclude by using the triangle inequality that

$$
\left\|\nabla\left(u^{m+1}-u^{m}\right)\right\| \leq\left\|\nabla\left(u_{h}^{m+1}-u_{h}^{m}\right)\right\|+\left\|\nabla\left(u^{m+1}-u_{h}^{m+1}\right)\right\|+\left\|\nabla\left(u^{m}-u_{h}^{m}\right)\right\| .
$$

A similar argument is used to evaluate $\int_{0}^{t_{1}}\left\|R_{v}(\cdot, s)\right\|_{H^{-1}(\Omega)} \mathrm{d} s$ and $\int_{t_{m}}^{t_{m+1}}\left\|R_{u}(\cdot, s)\right\| \mathrm{d} s$. Combining all these bounds leads to the desired result.

The same arguments yield that estimate (5.3) still holds with $t_{n+1}$ replaced by any $t$ in $] t_{n}, t_{n+1}[$. Further estimates seem necessary, in order to compare the indicator with the error.

Corollary 5.2. The following a posteriori error estimates hold between the solution $u$ of problem (2.1) and the solution $\left(u^{n}\right)_{0 \leq n \leq N}$ of problem (3.1), for all $n, 1 \leq n \leq N-1$ :

$$
\begin{array}{r}
\left\|\int_{t_{n}}^{t_{n+1}} \nabla\left(u-u_{\tau}\right)(s) \mathrm{d} s\right\| \leq \sum_{k=0}^{1}\left\|\partial_{t} u\left(t_{n+1-k}\right)-\frac{u^{n+1-k}-u^{n-k}}{\tau_{n-k}}\right\|_{H^{-1}(\Omega)} \\
+\eta_{n}+\tau_{n} \sum_{k=0}^{1}\left\|\nabla\left(u^{n+1-k}-u_{h}^{n+1-k}\right)\right\|,
\end{array}
$$

and

$$
\begin{aligned}
\left\|\int_{t_{n}}^{t_{n+1}}\left(v-v_{\tau}\right)(s) \mathrm{d} s\right\| & \leq \sum_{k=0}^{1}\left\|u\left(t_{n+1-k}\right)-u^{n+1-k}\right\| \\
& +\eta_{n}+\tau_{n} \sum_{k=0}^{1}\left\|\frac{\left(u^{n+1-k}-u_{h}^{n+1-k}\right)-\left(u^{n-k}-\Pi_{n+1-k, h} u_{h}^{n-k}\right)}{\tau_{n-k}}\right\| .
\end{aligned}
$$

Proof: Integrating the second line of the residual equation from (5.2) gives

$$
-\int_{t_{n}}^{t_{n+1}} \Delta\left(u-u_{\tau}\right)(s) \mathrm{d} s=\int_{t_{n}}^{t_{n+1}} R_{v}(s) \mathrm{d} s-\left(v-v_{\tau}\right)\left(t_{n+1}\right)+\left(v-v_{\tau}\right)\left(t_{n}\right),
$$

and taking the $H^{-1}(\Omega)$-norm of the left-hand side thus leads to

$$
\begin{aligned}
\| \int_{t_{n}}^{t_{n+1}} & \Delta\left(u-u_{\tau}\right)(s) \mathrm{d} s \|_{H^{-1}(\Omega)} \\
& \quad \leq\left\|\int_{t_{n}}^{t_{n+1}} R_{v}(s) \mathrm{d} s\right\|_{H^{-1}(\Omega)}+\left\|\left(v-v_{\tau}\right)\left(t_{n+1}\right)\right\|_{H^{-1}(\Omega)}+\left\|\left(v-v_{\tau}\right)\left(t_{n}\right)\right\|_{H^{-1}(\Omega)} .
\end{aligned}
$$

To evaluate the first term, we write

$$
\begin{aligned}
\left\|\int_{t_{n}}^{t_{n+1}} \Delta\left(u-u_{\tau}\right)(s) \mathrm{d} s\right\|_{H^{-1}(\Omega)}=\sup _{w \in H_{0}^{1}(\Omega)} \frac{\left(\int_{t_{n}}^{t_{n+1}} \Delta\left(u-u_{\tau}\right)(s) \mathrm{d} s, w\right)}{\|\nabla w\|} & =\sup _{w \in H_{0}^{1}(\Omega)} \frac{-\left(\int_{t_{n}}^{t_{n+1}}\left(\nabla\left(u-u_{\tau}\right)(s) \mathrm{d} s, \nabla w\right)\right.}{\|\nabla w\|} .
\end{aligned}
$$


On taking $w$ equal to $-\int_{t_{n}}^{t_{n+1}}\left(u-u_{\tau}\right)(s) \mathrm{d} s$, we obtain that

$$
\left\|\int_{t_{n}}^{t_{n+1}} \Delta\left(u-u_{\tau}\right)(s) \mathrm{d} s\right\|_{H^{-1}(\Omega)} \geq\left\|\int_{t_{n}}^{t_{n+1}} \nabla\left(u-u_{\tau}\right)(s) \mathrm{d} s\right\| .
$$

We note in passing that the two sides of the last inequality are, in fact, equal; this can be seen by applying the Cauchy-Schwarz inequality in the numerator of the right-most expression in the penultimate chain of equalities. Similar but simpler arguments (since $R_{v}$ is piecewise affine with respect to $t$ ) lead to the bound

$$
\left\|\int_{t_{n}}^{t_{n+1}} R_{v}(s) \mathrm{d} s\right\|_{H^{-1}(\Omega)} \leq \tau_{n}\left\|\nabla\left(u^{n+1}-u^{n}\right)\right\| .
$$

We conclude the proof of (5.4) by using the same triangle inequality as in the proof of Proposition 5.1. We skip the proof of (5.5) since it relies on exactly the same arguments (integrating the first line of equation (5.2) and taking the $L^{2}(\Omega)$-norm of the left-hand side).

On adding estimate (5.4) to (5.5) and using (5.3) in the right-hand side of the resulting inequality, we deduce that

$$
\left\|\int_{t_{n}}^{t_{n+1}} \nabla\left(u-u_{\tau}\right)(s) \mathrm{d} s\right\|+\left\|\int_{t_{n}}^{t_{n+1}}\left(v-v_{\tau}\right)(s) \mathrm{d} s\right\|
$$

is bounded by the right-hand side of (5.3). Next, we prove an upper bound on the refinement indicators $\eta_{n}, 1 \leq n \leq N-1$.

Proposition 5.3. The following estimate holds for each indicator $\eta_{n}$ defined in (5.1), $1 \leq n \leq N-1$ :

$$
\begin{aligned}
\eta_{n} \leq \sum_{k=0}^{1}\left\|\left(\partial_{t} u\right)\left(t_{n+1-k}\right)-\frac{u^{n+1-k}-u^{n-k}}{\tau_{n-k}}\right\|_{H^{-1}(\Omega)}+\left\|u\left(t_{n+1-k}\right)-u^{n+1-k}\right\| \\
+\left\|\int_{t_{n}}^{t_{n+1}} \nabla\left(u-u_{\tau}\right)(s) \mathrm{d} s\right\|+\left\|\int_{t_{n}}^{t_{n+1}}\left(v-v_{\tau}\right)(s) \mathrm{d} s\right\| \\
+\tau_{n} \sum_{k=0}^{1}\left\|\nabla\left(u^{n+1-k}-u_{h}^{n+1-k}\right)\right\| \\
\quad+\tau_{n} \sum_{k=0}^{1}\left\|\frac{\left(u^{n+1-k}-u_{h}^{n+1-k}\right)-\left(u^{n-k}-\Pi_{n+1-k, h} u_{h}^{n-k}\right)}{\tau_{n-k}}\right\| .
\end{aligned}
$$

Proof: We first note, by using triangle inequalities analogous to those in the proof of Proposition 5.1, that it suffices to bound the two quantities

$$
\tau_{n}\left\|\nabla\left(u^{n+1}-u^{n}\right)\right\|, \quad \tau_{n}\left\|v^{n+1}-v^{n}\right\| .
$$


1) To estimate the first of these, we take the inner product of the second line of (5.2) with $u^{n+1}-u^{n}$ and integrate between $t_{n}$ and $t_{n+1}$; this gives

$$
\begin{aligned}
\frac{\tau_{n}}{2}\left\|\nabla\left(u^{n+1}-u^{n}\right)\right\|^{2} \leq \int_{t_{n}}^{t_{n+1}}\left(\partial_{t}\left(v-v_{\tau}\right)(s), u^{n+1}-u^{n}\right) \mathrm{d} s & \\
& +\left(\int_{t_{n}}^{t_{n+1}} \nabla\left(u-u_{\tau}\right)(s) \mathrm{d} s, \nabla\left(u^{n+1}-u^{n}\right)\right) .
\end{aligned}
$$

Integrating by parts in the first term of the right-hand side yields

$$
\begin{aligned}
& \frac{\tau_{n}}{2}\left\|\nabla\left(u^{n+1}-u^{n}\right)\right\|^{2} \\
& \leq\left(\left(\partial_{t} u\right)\left(t_{n+1}\right)-\frac{u^{n+1}-u^{n}}{\tau_{n}}, u^{n+1}-u^{n}\right)-\left(\left(\partial_{t} u\right)\left(t_{n}\right)-\frac{u^{n}-u^{n-1}}{\tau_{n-1}}, u^{n+1}-u^{n}\right) \\
& \quad+\left(\int_{t_{n}}^{t_{n+1}} \nabla\left(u-u_{\tau}\right)(s) \mathrm{d} s, \nabla\left(u^{n+1}-u^{n}\right)\right) .
\end{aligned}
$$

Hence, by a simple application of the Cauchy-Schwarz inequality, we get

$$
\begin{gathered}
\frac{\tau_{n}}{2}\left\|\nabla\left(u^{n+1}-u^{n}\right)\right\| \\
\leq\left\|\left(\partial_{t} u\right)\left(t_{n+1}\right)-\frac{u^{n+1}-u^{n}}{\tau_{n}}\right\|_{H^{-1}(\Omega)}+\left\|\left(\partial_{t} u\right)\left(t_{n}\right)-\frac{u^{n}-u^{n-1}}{\tau_{n-1}}\right\|_{H^{-1}(\Omega)} \\
\quad+\left\|\int_{t_{n}}^{t_{n+1}} \nabla\left(u-u_{\tau}\right)(s) \mathrm{d} s\right\| .
\end{gathered}
$$

2) Similarly, to evaluate the second quantity, we take the inner product of the first line of (5.2) with $v^{n+1}-v^{n}$ and integrate between $t_{n}$ and $t_{n+1}$. This leads to

$$
\begin{aligned}
\frac{\tau_{n}}{2}\left\|v^{n+1}-v^{n}\right\|^{2} \leq\left(u\left(t_{n+1}\right)-u^{n+1}, v^{n+1}-v^{n}\right)- & \left(u\left(t_{n}\right)-u^{n}, v^{n+1}-v^{n}\right) \\
& -\left(\int_{t_{n}}^{t_{n+1}}\left(v-v_{\tau}\right)(s) \mathrm{d} s, v^{n+1}-v^{n}\right) .
\end{aligned}
$$

We conclude the argument in the same manner as was discussed previously.

\section{THE SPACE DISCRETIZATION}

For each $K$ in $\mathcal{T}_{n, h}$, we introduce the set $\mathcal{E}_{K}$ of endpoints in dimension $d=1$, edges in dimension $d=2$ or faces in dimension $d=3$ of $K$ which are not contained in $\partial \Omega$. In dimension $d \geq 2$, for each $e$ in $\mathcal{E}_{K}$, we use the following notation:

- $h_{e}$ denotes the length of $e$ in dimension $d=2$ or the diameter of $e$ in dimension $d=3$;

- $\boldsymbol{n}_{e}$ denotes the unit outward normal vector to $K$ on $e$;

- $[\cdot]_{e}$ denotes the jump through $e$ in the direction of $\boldsymbol{n}_{e}$.

For each $n, 1 \leq n \leq N-1$, and each $K$ in $\mathcal{T}_{n+1, h}$, we consider the refinement indicator defined by

$$
\begin{array}{r}
\eta_{n, K}=\left\|u_{h}^{n}-\Pi_{n+1, h} u_{h}^{n}\right\|_{L^{2}(K)}+h_{K}\left\|\frac{u_{h}^{n+1}-}{\Pi_{n+1, h} u_{h}^{n}}-\frac{u_{h}^{n}-\Pi_{n, h} u_{h}^{n-1}}{\tau_{n-1}}\right\|_{L^{2}(K)} \\
+\chi_{d} \tau_{n} \sum_{e \in \mathcal{E}_{K}} h_{e}^{\frac{1}{2}}\left\|\left[\partial_{n_{e}} u_{h}^{n+1}\right]_{e}\right\|_{L^{2}(e)},
\end{array}
$$


where $\chi_{d}$ is equal to zero in dimension $d=1$ and to 1 in dimension $d \geq 2$. Note that the first term in this definition stems from the use of different spatial meshes on the various time levels, while the other two terms are the same as those in standard residual-based error bounds for the elliptic equation (4.5), see $[20, \S 1.2]$.

We now consider the residual equation associated with problem (4.3) - (4.4). For $1 \leq n \leq N$, define

$$
v^{n}=\frac{u^{n}-u^{n-1}}{\tau_{n-1}}, \quad v_{h}^{n}=\frac{u_{h}^{n}-\Pi_{n, h} u_{h}^{n-1}}{\tau_{n-1}}, \quad e_{h u}^{n}=u^{n}-u_{h}^{n}, \quad e_{h v}^{n}=v^{n}-v_{h}^{n} .
$$

It then follows from (3.1) and (4.4) that the vector error $E_{h}^{n}=\left(\begin{array}{c}e_{h u}^{n} \\ e_{h v}^{n}\end{array}\right)$ satisfies the residual system

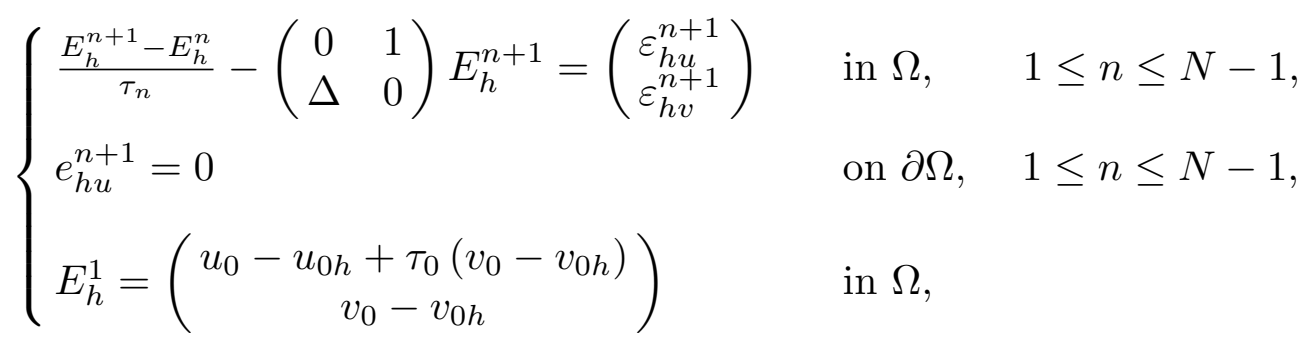

where the quantities $\varepsilon_{h u}^{n+1}$ and $\varepsilon_{h u}^{n+1}$ are defined in $H^{-1}(\Omega)$ by

$$
\begin{aligned}
& \left\langle\varepsilon_{h u}^{n+1}, v\right\rangle=\left(\frac{u_{h}^{n}-\Pi_{n+1, h} u_{h}^{n}}{\tau_{n}}, v\right), \\
& \left\langle\varepsilon_{h v}^{n+1}, v\right\rangle=-\frac{1}{\tau_{n}}\left(\frac{u_{h}^{n+1}-\Pi_{n+1, h} u_{h}^{n}}{\tau_{n}}-\frac{u_{h}^{n}-\Pi_{n, h} u_{h}^{n-1}}{\tau_{n-1}}, v\right)-\left(\nabla u_{h}^{n+1}, \nabla v\right) .
\end{aligned}
$$

The proof of the a posteriori error estimate stated in the next proposition relies on system $(5.9)$.

Proposition 5.4. The following a posteriori error estimate holds between the solutions $\left(u^{n}\right)_{0 \leq n \leq N}$ of problem (3.1) and $\left(u_{h}^{n}\right)_{0 \leq n \leq N}$ of problem $(4.3)-(4.4)$, for all $n, 1 \leq n \leq$ $N-1$ :

$$
\begin{aligned}
& \left\|\frac{\left(u^{n+1}-u_{h}^{n+1}\right)-\left(u^{n}-\Pi_{n+1, h} u_{h}^{n}\right)}{\tau_{n}}\right\|_{H^{-1}(\Omega)}+\left\|u^{n+1}-u_{h}^{n+1}\right\| \\
& \leq c\left(\sum_{m=1}^{n}\left(\sum_{K \in \mathcal{T}_{m+1, h}} \eta_{m, K}^{2}\right)^{\frac{1}{2}}\right. \\
& \left.\quad+\left\|u_{0}-u_{0 h}\right\|+\tau_{0}\left\|v_{0}-v_{0 h}\right\|+\left\|v_{0}-v_{0 h}\right\|_{H^{-1}(\Omega)}\right) .
\end{aligned}
$$

Proof: Applying estimate (3.10) to system (5.9) and then taking the square-root of both sides of the resulting inequality and using that, for any two nonnegative real numbers $a$ and $b, \frac{1}{\sqrt{2}}(a+b) \leq \sqrt{a^{2}+b^{2}} \leq a+b$, we obtain that

$$
\left\|e_{h v}^{n+1}\right\|_{H^{-1}(\Omega)}+\left\|e_{h u}^{n+1}\right\| \leq c\left(\left\|e_{h v}^{1}\right\|_{H^{-1}(\Omega)}+\left\|e_{h u}^{1}\right\|+\sum_{m=1}^{n} \tau_{m}\left(\left\|\varepsilon_{h v}^{m+1}\right\|_{H^{-1}(\Omega)}+\left\|\varepsilon_{h u}^{m+1}\right\|\right)\right) .
$$


Now, the terms on the left-hand side of this inequality are exactly the same as in (5.11), so we now evaluate the quantities on the right-hand side.

1) The bounds on $\left\|e_{h v}^{1}\right\|_{H^{-1}(\Omega)}$ and $\left\|e_{h u}^{1}\right\|$ come from the third equation in (5.9); hence the last three terms on the right-hand side of (5.11).

2) It follows from the definition of $\varepsilon_{h u}^{m+1}$ that

$$
\left\|\varepsilon_{h u}^{m+1}\right\|=\left\|\frac{u_{h}^{m}-\Pi_{m+1, h} u_{h}^{m}}{\tau_{m}}\right\|=\tau_{m}^{-1}\left(\sum_{K \in \mathcal{T}_{m+1, h}}\left\|u_{h}^{m}-\Pi_{m+1, h} u_{h}^{m}\right\|_{L^{2}(K)}^{2}\right)^{\frac{1}{2}} .
$$

3) We have

$$
\left\|\varepsilon_{h v}^{m+1}\right\|_{H^{-1}(\Omega)}=\sup _{v \in H_{0}^{1}(\Omega)} \frac{\left\langle\varepsilon_{h v}^{m+1}, v\right\rangle}{\|\nabla v\|} .
$$

We deduce from problem (4.4) that $\left\langle\varepsilon_{h v}^{m+1}, v_{h}\right\rangle=0$ for all $v_{h} \in X_{m+1, h}$; therefore, for any $v$ in $H_{0}^{1}(\Omega)$ and $v_{h}$ in $X_{m+1, h}$,

$$
\left\langle\varepsilon_{h v}^{m+1}, v\right\rangle=-\frac{1}{\tau_{m}}\left(\frac{u_{h}^{m+1}-\Pi_{m+1, h} u_{h}^{m}}{\tau_{m}}-\frac{u_{h}^{m}-\Pi_{m, h} u_{h}^{m-1}}{\tau_{m-1}}, v-v_{h}\right)-\left(\nabla u_{h}^{m+1}, \nabla\left(v-v_{h}\right)\right) .
$$

Integrating by parts in the last term on each $K$ of $\mathcal{T}_{m+1, h}$ and recalling that $u_{h}^{m+1}$ is affine on $K$ leads, with an obvious modification in dimension $d=1$, to the equality

$$
\begin{aligned}
\left\langle\varepsilon_{h v}^{m+1}, v\right\rangle=-\frac{1}{\tau_{m}}\left(\frac{u_{h}^{m+1}-\Pi_{m+1, h} u_{h}^{m}}{\tau_{m}}-\frac{u_{h}^{m}-\Pi_{m, h} u_{h}^{m-1}}{\tau_{m-1}}, v-v_{h}\right) & \left.-\frac{1}{2} \sum_{K \in \mathcal{T}_{m+1, h}} \sum_{e \in \mathcal{E}_{K}} \int_{e}\left[\partial_{n_{e}} u_{h}^{m+1}\right]_{e}(\tau)\left(v-v_{h}\right)(\tau) d \tau\right) .
\end{aligned}
$$

In dimension $d=1$, since $H^{1}(\Omega)$ is contained in the space of continuous functions, we take $v_{h}$ equal to the continuous piecewise linear Lagrange interpolant of $v$, where the interpolation points are the endpoints of the intervals $K$ of $\mathcal{T}_{m+1, h}$; hence, the last term then disappears. In dimension $d \geq 2$, we take $v_{h}$ equal to the image of $v$ under the operator $\mathcal{R}_{m+1, h}$ introduced in Section 4, and we use (4.7) and the further estimate, see [8, Chap. IX, Cor. 3.12]),

$$
\left\|v-\mathcal{R}_{m+1, h} v\right\|_{L^{2}(e)} \leq c h_{e}^{\frac{1}{2}}\|v\|_{H^{1}\left(\Delta_{K}\right)} .
$$

These give

$$
\begin{array}{r}
\left\langle\varepsilon_{h v}^{m+1}, v\right\rangle \leq \sum_{K \in \mathcal{T}_{m+1, h}}\left(\frac{h_{K}}{\tau_{m}}\left\|\frac{u_{h}^{m+1}-\Pi_{m+1, h} u_{h}^{m}}{\tau_{m}}-\frac{u_{h}^{m}-\Pi_{m, h} u_{h}^{m-1}}{\tau_{m-1}}\right\|_{L^{2}(K)}\right. \\
\left.+\chi_{d} \sum_{e \in \mathcal{E}_{K}} h_{e}^{\frac{1}{2}}\left\|\left[\partial_{n_{e}} u_{h}^{m+1}\right]_{e}\right\|_{L^{2}(e)}\right)\|v\|_{H^{1}\left(\Delta_{K}\right)} .
\end{array}
$$

We conclude by using a Cauchy-Schwarz inequality for finite sums and recalling that each element in $\mathcal{T}_{m+1, h}$ is contained in a finite number of $\Delta_{K}$, where "finite" means bounded as a function of the regularity parameter $\sigma$ of the family of triangulations. That completes the proof of (5.11). 
We also need a bound on the other terms which appear in (5.3).

Corollary 5.5. The following a posteriori error estimate holds between the solutions $\left(u^{n}\right)_{0 \leq n \leq N}$ of problem (3.1) and $\left(u_{h}^{n}\right)_{0 \leq n \leq N}$ of problem (4.3) - (4.4), for all $n, 1 \leq n \leq$ $N-1$ :

$$
\begin{aligned}
& \tau_{n}\left(\left\|\nabla\left(u^{n+1}-u_{h}^{n+1}\right)\right\|\right.\left.+\left\|\frac{\left(u^{n+1}-u_{h}^{n+1}\right)-\left(u^{n}-\Pi_{n+1, h} u_{h}^{n}\right)}{\tau_{n}}\right\|\right) \\
& \leq c\left(\sum_{m=1}^{n}\left(\sum_{K \in \mathcal{T}_{m+1, h}} \eta_{m, K}^{2}\right)^{\frac{1}{2}}\right. \\
&\left.\quad+\left\|u_{0}-u_{0 h}\right\|+\tau_{0}\left\|v_{0}-v_{0 h}\right\|+\left\|v_{0}-v_{0 h}\right\|_{H^{-1}(\Omega)}\right) .
\end{aligned}
$$

Proof: Taking the $H^{-1}(\Omega)$ norm of the second line of the residual equation in (5.9) gives

$$
\left\|\nabla\left(u^{n+1}-u_{h}^{n+1}\right)\right\| \leq \frac{1}{\tau_{n}}\left(\left\|e_{h v}^{n+1}\right\|_{H^{-1}(\Omega)}+\left\|e_{h v}^{n}\right\|_{H^{-1}(\Omega)}\right)+\left\|\varepsilon_{h v}^{n+1}\right\|_{H^{-1}(\Omega)} .
$$

Multiplying by $\tau_{n}$ and using (5.11) gives the estimate for the first term. Similarly, taking the $L^{2}(\Omega)$-norm of the first line in $(5.9)$ yields

$$
\left\|v^{n}-v_{h}^{n}\right\| \leq \frac{1}{\tau_{n}}\left(\left\|e_{h u}^{n+1}\right\|+\left\|e_{h u}^{n}\right\|\right)+\left\|\varepsilon_{h u}^{n+1}\right\|,
$$

and the same arguments as previously lead to the conclusion.

Of course, the quantity

$$
\tau_{n}\left(\left\|\nabla\left(u^{n}-u_{h}^{n}\right)\right\|+\left\|\frac{\left(u^{n}-u_{h}^{n}\right)-\left(u^{n-1}-\Pi_{n, h} u_{h}^{n-1}\right)}{\tau_{n-1}}\right\|\right),
$$

which also appears in (5.3), is bounded similarly by using the regularity parameter

$$
\sigma_{\tau}=\max _{1 \leq m \leq N-1} \frac{\tau_{m}}{\tau_{m-1}}
$$

which is most often bounded independently of the discretization. In the sequel, we shall always suppose that this is the case.

In order to prove an upper bound for each indicator $\eta_{n, K}$, we are lead to make the following assumption, which is not at all restrictive in the context of mesh adaptivity.

Assumption 5.6. For $1 \leq n \leq N-1$, there exists a regular family of triangulations $\left(\mathcal{T}_{n+1, h}^{*}\right)_{h}$ such that, for all $h$ and $n, 1 \leq n \leq N$, each element of $\mathcal{T}_{n, h}$ and $\mathcal{T}_{n+1, h}$ is the union of at most $s$ elements of $\mathcal{T}_{n+1, h}^{*}$ (where $s$ is bounded independently of $h$ and $n$ ). 
Proposition 5.7. If Assumption 5.6 holds, then the following estimate is valid for each indicator $\eta_{n, K}$ defined in (5.7), for all $K$ in $\mathcal{T}_{n+1, h}, 1 \leq n \leq N-1$ :

$$
\begin{gathered}
\eta_{n, K} \leq c \sum_{k=0}^{1}\left(\left\|\frac{\left(u^{n+1-k}-u_{h}^{n+1-k}\right)-\left(u^{n-k}-\Pi_{n+1-k, h} u_{h}^{n-k}\right)}{\tau_{n}}\right\|_{H^{-1}\left(\omega_{K}\right)}\right. \\
\left.+\left\|u^{n+1-k}-u_{h}^{n+1-k}\right\|_{L^{2}(K)}\right) \\
+\tau_{n}\left(\left\|\frac{\left(u^{n+1}-u_{h}^{n+1}\right)-\left(u^{n}-\Pi_{n+1, h} u_{h}^{n}\right)}{\tau_{n}}\right\|_{L^{2}(K)}\right. \\
\left.\left.+\left\|\nabla\left(u^{n+1}-u_{h}^{n+1}\right)\right\|_{L^{2}\left(\omega_{K}\right)^{d}}\right)\right),
\end{gathered}
$$

where $\omega_{K}$ is equal to $K$ in dimension $d=1$ and denotes the union of elements of $\mathcal{T}_{n+1, h}$ that share at least an edge with $K$ in dimension $d=2$ or a face with $K$ in dimension $d=3$.

Proof: We bound successively the three terms in $\eta_{n, K}$, which we denote by $\eta_{n, K}^{(1)}, \eta_{n, K}^{(2)}$ and $\eta_{n, K}^{(3)}$.

1) The first line in $(5.9)$ can equivalently be written

$$
\frac{e_{h u}^{n+1}-e_{h u}^{n}}{\tau_{n}}-e_{h v}^{n+1}=\varepsilon_{n u}^{n+1}=\frac{u_{h}^{n}-\Pi_{n+1, h} u_{h}^{n}}{\tau_{n}} .
$$

Hence, taking the $L^{2}(K)$-norm of this equation and multiplying by $\tau_{n}$ gives

$$
\begin{aligned}
\eta_{n, K}^{(1)} \leq \sum_{k=0}^{1}\left\|u^{n+1-k}-u_{h}^{n+1-k}\right\|_{L^{2}(K)} & \\
& \quad+\tau_{n}\left\|\frac{\left(u^{n+1}-u_{h}^{n+1}\right)-\left(u^{n}-\Pi_{n+1, h} u_{h}^{n}\right)}{\tau_{n}}\right\|_{L^{2}(K)} .
\end{aligned}
$$

2) Let $v_{K}$ be the function defined by

$$
v_{K}= \begin{cases}\left(\frac{u_{h}^{n+1}-\Pi_{n+1, h} u_{h}^{n}}{\tau_{n}}-\frac{u_{h}^{n}-\Pi_{n, h} u_{h}^{n-1}}{\tau_{n-1}}\right) \psi_{K} & \text { on } K, \\ 0 & \text { on } \Omega \backslash K,\end{cases}
$$

where $\psi_{K}$ denotes the bubble function on $K$, equal to the product of the $d+1$ barycentric coordinates on $K$. It is readily checked by integration by parts on $K$ that

$$
\left\langle\varepsilon_{h v}^{n+1}, v_{K}\right\rangle=-\frac{1}{\tau_{n}}\left\|\left(\frac{u_{h}^{n+1}-\Pi_{n+1, h} u_{h}^{n}}{\tau_{n}}-\frac{u_{h}^{n}-\Pi_{n, h} u_{h}^{n-1}}{\tau_{n-1}}\right) \psi_{K}^{\frac{1}{2}}\right\|_{L^{2}(K)}^{2} .
$$

Hence, taking the inner product of the second equation of the residual system (5.9) with $-\tau_{n} v_{K}$ gives

$$
\begin{aligned}
&\left\|\left(\frac{u_{h}^{n+1}-\Pi_{n+1, h} u_{h}^{n}}{\tau_{n}}-\frac{u_{h}^{n}-\Pi_{n, h} u_{h}^{n-1}}{\tau_{n-1}}\right) \psi_{K}^{\frac{1}{2}}\right\|_{L^{2}(K)}^{2} \\
& \leq\left(\sum_{k=0}^{1}\left\|\frac{\left(u^{n+1-k}-u_{h}^{n+1-k}\right)-\left(u^{n-k}-\Pi_{n+1-k, h} u_{h}^{n-k}\right)}{\tau_{n}}\right\|_{H^{-1}(K)}\right. \\
&\left.\quad+\tau_{n}\left\|\nabla\left(u^{n+1}-u_{h}^{n+1}\right)\right\|_{L^{2}(K)^{d}}\right)\left\|\nabla v_{K}\right\|_{L^{2}(K)^{d}} .
\end{aligned}
$$


We now use the following standard inverse inequality, on each element $\kappa$ of $\mathcal{T}_{n+1, h}^{*}$ contained in $K$ (note that the restriction of $v_{K}$ to $\kappa$ is a polynomial of degree $\leq d+2$ ):

$$
\left\|\nabla v_{K}\right\|_{L^{2}(\kappa)^{d}} \leq c h_{\kappa}^{-1}\left\|v_{K}\right\|_{L^{2}(\kappa)},
$$

where the diameter $h_{\kappa}$ of $\kappa$ is larger than $c h_{K}$, for a constant $c$ only depending on the parameter $s$ introduced in Assumption 5.6. We also have the inequality [20, Lemma 3.3]

$$
\forall w \in \mathcal{P}_{1}(\kappa), \quad\left\|w \psi_{K}\right\|_{L^{2}(\kappa)} \leq\|w\|_{L^{2}(\kappa)} \leq c\left\|w \psi_{K}^{\frac{1}{2}}\right\|_{L^{2}(\kappa)} .
$$

Combining all this leads to the estimate

$$
\begin{array}{r}
\eta_{n, K}^{(2)} \leq c\left(\sum_{k=0}^{1}\left\|\frac{\left(u^{n+1-k}-u_{h}^{n+1-k}\right)-\left(u^{n-k}-\Pi_{n+1-k, h} u_{h}^{n-k}\right)}{\tau_{n}}\right\|_{H^{-1}(K)}\right. \\
\left.+\tau_{n}\left\|\nabla\left(u^{n+1}-u_{h}^{n+1}\right)\right\|_{L^{2}(K)^{d}}\right) .
\end{array}
$$

3) Finally, in dimension $d \geq 2$, for any $e$ in $\mathcal{E}_{K}$, let $K^{\prime}$ denote the other element of $\mathcal{T}_{n+1, h}$ such that $e$ is contained in $\partial K^{\prime}$. We now take the inner product of the second equation of the residual system (5.9) with $-\tau_{n} v_{e}$, the function $v_{e}$ being defined by

$$
v_{e}= \begin{cases}\mathcal{L}_{e, K}\left(\left[\partial_{n_{e}} u_{h}^{n+1}\right]_{e} \psi_{e}\right) & \text { on } K, \\ \mathcal{L}_{e, K^{\prime}}\left(\left[\partial_{n_{e}} u_{h}^{n+1}\right]_{e} \psi_{e}\right) & \text { on } K^{\prime}, \\ 0 & \text { on } \Omega \backslash\left(K \cup K^{\prime}\right),\end{cases}
$$

where $\psi_{e}$ stands for the bubble function on $e$. Here, $\mathcal{L}_{e, K}$ and $\mathcal{L}_{e, K^{\prime}}$ denote lifting operators from polynomials on $e$ vanishing on $\partial e$ into polynomials on $K$ vanishing on $\partial K \backslash e$ and into polynomials on $K^{\prime}$ vanishing on $\partial K^{\prime} \backslash e$, respectively; both are constructed by affine transformation from a fixed lifting operator on a reference element $\hat{K}$. Then, the same arguments as previously (which we skip for the sake of brevity) together with the inequality

$$
\left\|\nabla \mathcal{L}_{e, K}\left(\left[\partial_{n_{e}} u_{h}^{n+1}\right]_{e} \psi_{e}\right)\right\|_{L^{2}(K)^{d}} \leq c h_{e}^{-\frac{1}{2}}\left\|\left[\partial_{n_{e}} u_{h}^{n+1}\right]_{e} \psi_{e}\right\|_{L^{2}(e)}
$$

and its analogue with $K$ replaced by $K^{\prime}$, lead to the estimate

$$
\begin{array}{r}
\eta_{n, K}^{(3)} \leq c\left(\sum_{K^{\prime} \subset \omega_{K}} \eta_{n, K^{\prime}}^{(2)}+\sum_{k=0}^{1}\left\|\frac{\left(u^{n+1-k}-u_{h}^{n+1-k}\right)-\left(u^{n-k}-\Pi_{n+1-k, h} u_{h}^{n-k}\right)}{\tau_{n}}\right\|_{H^{-1}\left(\omega_{K}\right)}\right. \\
\left.+\tau_{n}\left\|\nabla\left(u^{n+1}-u_{h}^{n+1}\right)\right\|_{L^{2}\left(\omega_{K}\right)^{d}}\right) .
\end{array}
$$

Combining estimates (5.15) to (5.17) and noting that, since extension by zero is a continuous (and therefore bounded) linear operator from $H_{0}^{1}(K)$ into $H_{0}^{1}\left(\omega_{K}\right)$, the norm $\|\cdot\|_{H^{-1}(K)}$ is smaller than the norm $\|\cdot\|_{H^{-1}\left(\omega_{K}\right)}$, we arrive at the desired result.

Since estimate (5.14) involves $H^{-1}\left(\omega_{K}\right)$-norms, it is not immediately obvious how to derive a global error bound from it. However, this can be achieved by multiplying the second line of (5.9) successively by

$$
\sum_{K \in \mathcal{T}_{n+1, h}} h_{K}^{2} v_{K} \quad \text { and } \quad \sum_{K \in \mathcal{T}_{n+1, h}} \sum_{e \in \mathcal{E}_{K}} h_{e} v_{e}
$$


where the functions $v_{K}$ and $v_{e}$ have been introduced in the previous proof. This leads us to our next result.

Corollary 5.8. Under Assumption 5.6, the following estimate holds for the family of indicators $\eta_{n, K}$ defined in (5.7), $1 \leq n \leq N-1$ :

$$
\begin{gathered}
\left(\sum_{K \in \mathcal{T}_{n+1, h}} \eta_{n, K}^{2}\right)^{\frac{1}{2}} \leq c \sum_{k=0}^{1}\left(\left\|\frac{\left(u^{n+1-k}-u_{h}^{n+1-k}\right)-\left(u^{n-k}-\Pi_{n+1-k, h} u_{h}^{n-k}\right)}{\tau_{n}}\right\|_{H^{-1}(\Omega)}\right. \\
\left.+\left\|u^{n+1-k}-u_{h}^{n+1-k}\right\|_{L^{2}(\Omega)}\right) \\
+\tau_{n}\left(\left\|\frac{\left(u^{n+1}-u_{h}^{n+1}\right)-\left(u^{n}-\Pi_{n+1, h} u_{h}^{n}\right)}{\tau_{n}}\right\|_{L^{2}(\Omega)}\right. \\
\left.\left.+\left\|\nabla\left(u^{n+1}-u_{h}^{n+1}\right)\right\|_{L^{2}(\Omega)^{d}}\right)\right) .
\end{gathered}
$$

Estimates (5.11) and (5.12), when compared with (5.18), prove the optimality of the a posteriori bounds with respect to the spatial discretisation parameter $h$ : indeed, up to terms which only involve the error in the approximation of the data $u_{0}$ and $v_{0}$, the global error at time $t_{n+1}$ is bounded by a constant times $\sum_{m=1}^{n} \eta_{m, h}$, where each $\eta_{n, h}$ denotes the Hilbertian sum of the refinement indicators at time $t_{n+1}$, namely

$$
\eta_{n, h}=\left(\sum_{K \in \mathcal{T}_{n+1, h}} \eta_{n, K}^{2}\right)^{\frac{1}{2}} .
$$

Conversely, the quantity $\sum_{m=1}^{n} \eta_{m, h}$ is bounded by a constant multiple of the sum, through times $t_{m+1}, 1 \leq m \leq n$, of the full-space-time errors. Finally we note that estimate (5.14) is local both in space and in time; hence, the $\eta_{n, K}$ provide an efficient tool for mesh adaptivity at each time $t_{n+1}$. As will be clear from our final result, there is a loss of optimality with respect to $\tau$ : the loss grows linearly with $n$, where $n$ is the counter of the time level $t_{n}$, so it is negligible at least for the first few time steps.

\section{A GLOBAL ERROR ESTIMATE}

When combining the estimates from Propositions 5.1 and 5.4 with Corollary 5.5, we obtain the following a posteriori bound for the global error.

Theorem 5.9. The following a posteriori error estimate holds between the solution $u$ of problem (2.1) and the solution $\left(u_{h}^{n}\right)_{0 \leq n \leq N}$ of problem (4.3) - (4.4), for all $t_{n+1}, 1 \leq n \leq$ $N-1$ :

$$
\begin{aligned}
& \left\|\left(\partial_{t} u\right)\left(t_{n+1}\right)-\frac{u_{h}^{n+1}-\Pi_{n+1, h} u_{h}^{n}}{\tau_{n}}\right\|_{H^{-1}(\Omega)}+\left\|u\left(t_{n+1}\right)-u_{h}^{n+1}\right\| \\
& \leq c\left(\sum_{m=1}^{n}\left(\eta_{m}+\left(1+\sigma_{\tau}\right)(n-m)\left(\sum_{K \in \mathcal{T}_{m+1, h}} \eta_{m, K}^{2}\right)^{\frac{1}{2}}\right)+\left(1+\sigma_{\tau}\right) n D_{\tau h}\right),
\end{aligned}
$$

where the term $D_{\tau h}$, given by

$$
D_{\tau h}=\tau_{0}\left\|\nabla u_{0}\right\|+\tau_{0}^{2}\left\|\nabla v_{0}\right\|+\left\|u_{0}-u_{0 h}\right\|+\tau_{0}\left\|v_{0}-v_{0 h}\right\|+\left\|v_{0}-v_{0 h}\right\|_{H^{-1}(\Omega)},
$$


only depends on the data.

\section{Conclusions and extensions.}

The error indicators that we have described in Section 5 allow for very natural adaptive strategies, both in space and in time. We only describe the simplest one. Let $\eta^{*}$ be a fixed tolerance.

Initialization: We first choose an initial time step $\tau_{0}$ such that

$$
\tau_{0}\left\|\nabla u_{0}\right\|+\tau_{0}^{2}\left\|\nabla v_{0}\right\| \leq \eta^{*}
$$

Indeed this term appears in (5.3). Next, we choose the triangulation $\mathcal{T}_{0, h}=\mathcal{T}_{1, h}$ such that

$$
\left\|u_{0}-u_{0 h}\right\|+\tau_{0}\left\|v_{0}-v_{0 h}\right\|+\left\|v_{0}-v_{0 h}\right\|_{H^{-1}(\Omega)} \leq \eta^{*}
$$

This term appears in (5.11). We then compute $u_{h}^{1}$.

Time adaptivity: Assuming that the time step $\tau_{n-1}$, the mesh $\mathcal{T}_{n, h}$ and the discrete solution $u_{h}^{n}$ are known, we first choose $\tau_{n}$ equal to $\tau_{n-1}$ and $\mathcal{T}_{n+1, h}$ equal to $\mathcal{T}_{n, h}$. We compute a first solution $u_{h}^{n+1}$ of problem (4.5) and the corresponding error indicator $\eta_{n}$ defined in (5.1). Next,

- if $\eta_{n}$ is smaller than $\eta^{*}$, we proceed to the spatial adaptivity step;

- if not, we divide $\tau_{n}$ by two (or by a constant times $\eta_{n} / \eta^{*}$ ) and perform a new computation.

Of course, this step can be iterated a number of times. This leads to the final value of $\tau_{n}$.

Spatial adaptivity: Assuming that the time step $\tau_{n}$ is known and that a first solution

$u_{h}^{n+1}$ has been computed on the mesh $\mathcal{T}_{n, h}$, we compute the indicators $\eta_{n, K}, K \in \mathcal{T}_{n, h}$, and their mean value $\bar{\eta}_{n}$. Then, we perform mesh adaptivity in the usual way:

- If $\eta_{n, K}$ is larger than $\bar{\eta}_{n}$, we cut $K$ into several elements (for instance 4 subelements in dimension $d=2$ by iteratively joining the midpoints of the edges of $K$ );

- If $\eta_{n, K}$ is much smaller than $\bar{\eta}_{n}$, we can also glue together some elements of the triangulation into a larger element.

We refer to $[14, \S 7.5 .1]$ for details on this procedure. This step can be iterated three or four times and the final mesh is called $\mathcal{T}_{n+1, h}$.

Even though the estimates proved in Section 5 do not imply the convergence of such an adaptive strategy, they give a good idea of its efficiency.

Finally, we note that the previous estimates can also be shown for more complex equations, including the variable-coefficient counterpart of the constant coefficient wave equation considered here, which arises from the modelling of wave-propagation in heterogeneous media, or for the time-dependent Maxwell system of electromagnetism. 


\section{References}

[1] M. Ainsworth, J.T. Oden - A Posteriori Error Estimation in Finite Element Analysis, J. Wiley \& Sons, New York (2000).

[2] S. Adjerid - A posteriori finite element error estimation for second-order hyperbolic problems, Comput. Methods Appl. Mech. Engrg. 191 (2002), 4699-4719.

[3] I. Babuška, T. Strouboulis - The Finite Element Method and Its Reliability, Oxford University Press, Oxford (2001).

[4] W. Bangerth - Mesh adaptivity and error control for a finite element approximation of the elastic wave equation. Mathematical and numerical aspects of wave propagation (Santiago de Compostela, 2000), 725-729, SIAM, Philadelphia, PA, (2000).

[5] W. Bangerth, R. Rannacher - Finite element approximation of the acoustic wave equation: error control and mesh adaptation, East-West J. Numer. Math. 7 (1999), 263-282.

[6] W. Bangerth, R. Rannacher - Adaptive finite element techniques for the acoustic wave equation, J. Comput. Acoust. 9 (2001), 575-591.

[7] A. Bergam, C. Bernardi, Z. Mghazli - A posteriori analysis of the finite element discretization of a nonlinear parabolic equation, to appear in Math. Comput.

[8] C. Bernardi, Y. Maday, F. Rapetti - Discrétisations variationnelles de problèmes aux limites elliptiques, Collection "Mathématiques et Applications" 45, Springer (2004).

[9] M. Bieterman, I. Babuška - The finite element method for parabolic equations. I. A posteriori error estimation, Numer. Math. 40 (1982), 339-371.

[10] M. Bieterman, I. Babuška - The finite element method for parabolic equations. II. A posteriori error estimation and adaptive approach, Numer. Math. 40 (1982), 373-406.

[11] P. Clément - Approximation by finite element functions using local regularization, R.A.I.R.O. Anal. Numér. 9 R2 (1975), 77-84.

[12] K. Eriksson, C. Johnson - Adaptive finite element methods for parabolic problems. I. A linear model problem, SIAM J. Numer. Anal. 28 (1991), 43-77.

[13] K. Eriksson, C. Johnson - Adaptive finite element methods for parabolic problems. IV. Nonlinear problems, SIAM J. Numer. Anal. 32 (1995), 1729-1749.

[14] P.J. Frey, P.-L. George — Maillages, applications aux éléments finis, Hermès, Paris (1999).

[15] E. Hairer, S.P. Nørsett, G. Wanner - Solving Ordinary Differential Equations. I. Nonstiff Problems, Springer Series in Comutational Mathematics 8, Springer-Verlag (1993).

[16] C. Johnson, Y.-Y. Nie, V. Thomée - An a posteriori error estimate and adaptive timestep control for a backward Euler discretization of a parabolic problem, SIAM J. Numer. Anal. 27 (1990), 277-291. 
[17] J.-L. Lions, E. Magenes — Problèmes aux limites non homogènes et applications, Dunod (1968).

[18] E. Süli - A posteriori error analysis and global error control for adaptive finite volume approximations of hyperbolic problems. Numerical Analysis 1995 (Dundee 1995), 169-190, Pitman Res. Notes Math. Ser. 344. Longman, Harlow (1996).

[19] E. Süli - A posteriori error analysis and adaptivity for finite element approximations of hyperbolic problems. In: D. Kröner, M. Ohlberger and C. Rohde (Eds.) An Introduction to Recent Developments in Theory and Numerics for Conservation Laws. Lecture Notes in Computational Science and Engineering Volume 5, 123-194, Springer-Verlag (1998).

[20] R. Verfürth - A Review of A Posteriori Error Estimation and Adaptive Mesh-Refinement Techniques, Wiley \& Teubner (1996).

[21] R. Verfürth - A posteriori error estimation techniques for non-linear elliptic and parabolic pdes, Revue européenne des éléments finis 9 (2000), 377-402. 